\title{
Development of a More Sustainable Appel Reaction
}

\author{
Andrew Jordan, ${ }^{*}$ Ross M. Denton, ${ }^{b}$ and Helen F. Sneddon ${ }^{c}$ \\ a GlaxoSmithKline Carbon Neutral Laboratory for Sustainable Chemistry, Jubilee Campus, University of \\ Nottingham, 6 Triumph Rd, Nottingham NG7 2GA. E-mail: andrew.jordan@nottingham.ac.uk. \\ ${ }^{b}$ GlaxoSmithKline Carbon Neutral Laboratory for Sustainable Chemistry, Jubilee Campus, University of \\ Nottingham, 6 Triumph Rd, Nottingham NG7 2GA. E-mail: ross.denton@nottingham.ac.uk. \\ c GSK, Medicines Research Centre, Gunnels Wood Road, Stevenage, Herts., UK, SG1 2NY. E-mail: \\ helen.f.sneddon@gsk.com; Fax: +44 (0)1438 768302; Tel: +44 (0)1438 763645
}

\section{Contents}

Development of a More Sustainable Appel Reaction

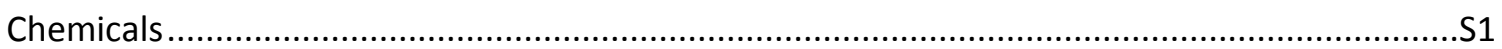

NMR

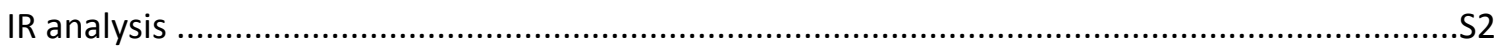

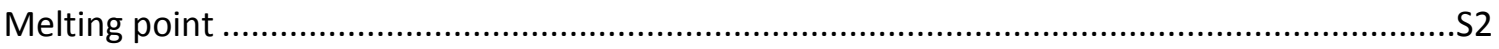

ESI-MS

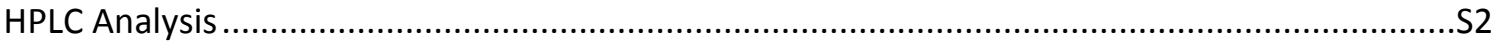

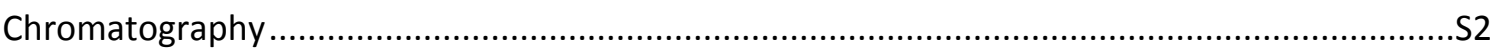

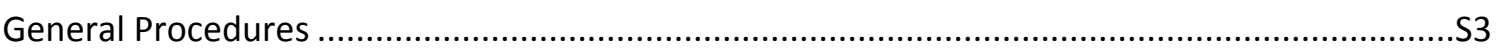

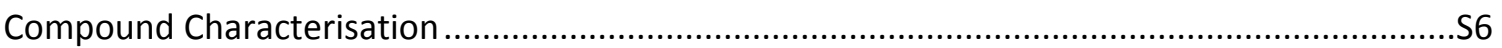

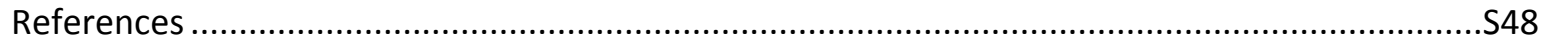

Number of Pages: 48

Number of Tables: 1

\section{Chemicals}

All chemicals used were purchased from Sigma-Aldrich, TCI Europe, Across Organics or Fluorochem. All reagents were used without further purification. Ethyl acetate and chloroform were dried over $3 \AA$ molecular sieves for 48 hours before use. All other solvents were from an Acro-seal Extra Dry 
preparation. Aluminium-backed Silicagel $60 \mathrm{~F}_{254}$ plates from E. Merck were used for thin layer chromatography.

\section{NMR}

The majority of NMR analysis was performed on a Bruker AC $400 \mathrm{MHz}$ spectrometer operating at 400 $\mathrm{MHz}$ for ${ }^{1} \mathrm{H}-\mathrm{NMR}$ and $101 \mathrm{MHz}$ for ${ }^{13} \mathrm{C}-\mathrm{NMR}$. Samples were run in deuterated chloroform $\left(\mathrm{CDCl}_{3}\right)$ or deuterated dimethyl sulfoxide (DMSO) where appropriate. A $500 \mathrm{MHz}$ Bruker spectrometer, operating at $500 \mathrm{MHz}$ for ${ }^{1} \mathrm{H}-\mathrm{NMR}$ and $126 \mathrm{MHz}$ for ${ }^{13} \mathrm{C}-\mathrm{NMR}$ was also used for analysis of some examples. All chemical shifts are reported in parts per million ( $\mathrm{ppm})$, are relative to the internal standard TMS and coupling constants $(J)$ are measured in Hertz $(\mathrm{Hz})$. Multiplicity is stated as follows: s-singlet, d-doublet, t-triplet, q-quartet, dd-doublet of doublets, dt-doublet of triplets, dq-doublet of quartets, tt-triplet of triplets, tq-triplet of quartets, ddd-doublet of doublet of doublets, m-multiplet, bs-broad singlet.

\section{IR analysis}

All IR analysis was carried out on a Bruker Alpha Platinum FT-IR spectrometer with ATR. For in situ IR experiments a REACT-IR 15 was used equipped with a diamond probe.

\section{Melting point}

Melting points were determined using a Stuart SMP20 melting point apparatus and the values are expressed in degrees Celsius $\left({ }^{\circ} \mathrm{C}\right)$. The parameters for the melting point analysis were set at $5{ }^{\circ} \mathrm{C}$ per minute ramp and melting point was determined manually. Melting points are uncorrected.

\section{ESI-MS}

High resolution mass spectrometry (HRMS) with accurate mass measurement to four decimal places was obtained for all new compounds described. Mass spectra were recorded in both positive and negative electrospray ionisation mode on a Bruker ESI MicroTOF mass spectrometer.

\section{HPLC Analysis}

HPLC for reaction monitoring used an Agilent 1200 system, with a standard gradient elution method: Waters XBridge $2.1 \times 30 \mathrm{~mm}$ column, stationary phase $\mathrm{C}_{18} 3.5 \mu \mathrm{m}, 0.8 \mathrm{~mL} / \mathrm{min}$ flow rate, detection at $254 \mathrm{~nm}$; elution with 5-95\% MeCN in 0.1\% aqueous TFA over 4 minutes.

\section{Chromatography}

A Biotage SP4 was used for the chromatographic purifications of compound $\mathbf{1 8}$ using a heptane:ethyl acetate gradient elution system with manual fraction collection. UV detector wavelength was set at 
$254 \mathrm{~nm}$. Interchim $30 \mu \mathrm{m}$ silica gel prepacked flash chromatography cartridges were used for this chromatographic separation.

\section{General Procedures}

\section{General Procedure 1 - Stoichiometric Chlorination}

To a room temperature stirred solution of triphenylphosphine oxide $(278 \mathrm{mg}, 1.0 \mathrm{mmol})$ in DMC (5 $\mathrm{mL}$ ) was added oxalyl chloride $(86 \mu \mathrm{L}, 1.0 \mathrm{mmol}$ ) Caution: exotherm and gas evolution. The reaction mixture was stirred for 15 minutes. The appropriate alcohol $(1.0 \mathrm{mmol})$ was then added dropwise to the reaction mixture over a 5 minute period. After the addition was complete the reaction was stirred for an additional 10 minutes. Solvent was removed in vacuo and the resulting crude mixture could be purified by either purification methods 1 or 2 . Vide infra.

\section{General Procedure 2 - Stoichiometric Bromination}

To a room temperature stirred solution of triphenylphosphine oxide $(278 \mathrm{mg}, 1.0 \mathrm{mmol})$ in DMC (5 $\mathrm{mL}$ ) was added an oxalyl bromide in DCM solution $(2 \mathrm{M})(0.5 \mathrm{~mL}, 1.0 \mathrm{mmol})$ Caution: exotherm and gas evolution. The reaction mixture was stirred for 15 minutes. The appropriate alcohol $(1.0 \mathrm{mmol})$ was then added dropwise to the reaction mixture over a 5 minute period. After the addition was complete the reaction was stirred for an additional 10 minutes. Solvent was removed in vacuo and the resulting crude mixture could be purified by either purification methods 1 or 2 . Vide infra.

\section{General Procedure 3 - Catalytic Chlorination}

To a room temperature stirred solution of triphenylphosphine oxide $(84 \mathrm{mg}, 0.30 \mathrm{mmol}$ ) in DMC (5 $\mathrm{mL}$ ) was added oxalyl chloride ( $26 \mu \mathrm{L}, 0.30 \mathrm{mmol}$ ) Caution: exotherm and gas evolution. The reaction mixture was stirred for 5 minutes. A $1 \mathrm{M}$ solution of the appropriate alcohol was prepared by dissolving $1 \mathrm{mmol}$ in $1 \mathrm{~mL}$ of DMC and loaded into syringe "A". Similarly $1.7 \mathrm{mmol}(147 \mu \mathrm{L})$ of $(\mathrm{COCl})_{2}$ was dissolved in $1 \mathrm{~mL}$ of DMC and loaded into syringe " $\mathrm{B}$ ". Both syringes A and B were then simultaneously added to the reaction mixture over a 7 hour period by syringe pump. Note: it is critical that both syringes are adequately primed with the appropriate solutions to ensure the absence of air bubbles. After addition completion, solvent was removed in vacuo and the resulting crude mixture could be purified by either Purification Methods 1 or 2 .

\section{General Procedure 4 - Catalytic Bromination with $(\mathrm{COBr})_{2}$}

To a room temperature stirred solution of triphenylphosphine oxide $(42 \mathrm{mg}, 0.15 \mathrm{mmol}$ ) in DMC (5 $\mathrm{mL}$ ) was added oxalyl bromide in dichloromethane (2M soln.) $(75 \mu \mathrm{L}, 0.15 \mathrm{mmol}$ ) Caution: exotherm and gas evolution. The reaction mixture was stirred for 5 minutes. A $1 \mathrm{M}$ solution of the appropriate 
alcohol was prepared by dissolving $1 \mathrm{mmol}$ in $1 \mathrm{~mL}$ of DMC and loaded into syringe " $\mathrm{A}$ ". Similarly 0.85 $\mathrm{mmol}$ of $(\mathrm{COBr})_{2}$ (2M in dichloromethane) $(425 \mu \mathrm{L})$ was diluted to $1 \mathrm{~mL}$ in DMC and loaded into syringe " $\mathrm{B}$ ". Both syringes $A$ and $B$ were then added to the reaction mixture over a 7 hour period by syringe pump. Note: it is critical that both syringes are adequately primed with the appropriate solutions to ensure no air bubbles. After addition completion, solvent was removed in vacuo and the resulting crude mixture could be purified by either purification methods 1 or 2 . Vide infra.

\section{General Procedure 5 - Catalytic Bromination with $(\mathrm{COCl})_{2}$ and $\mathrm{LiBr}$}

To a room temperature stirred solution of triphenylphosphine oxide $(84 \mathrm{mg}, 0.30 \mathrm{mmol}$ ) in DMC (5 $\mathrm{mL}$ ) was added oxalyl chloride $(26 \mu \mathrm{L}, 0.30 \mathrm{mmol})$ and $\mathrm{LiBr}(260 \mathrm{mg}, 3.0 \mathrm{mmol})$ Caution: exotherm and gas evolution. The reaction mixture was stirred for 5 minutes. A $1 \mathrm{M}$ solution of the appropriate alcohol was prepared by dissolving $1 \mathrm{mmol}$ in $1 \mathrm{~mL}$ of DMC and loading into syringe " $\mathrm{A}$ ". Similarly 1.7 $\mathrm{mmol}(147 \mu \mathrm{L})$ of $(\mathrm{COCl})_{2}$ was dissolved in $1 \mathrm{~mL}$ of DMC and loaded into syringe " $\mathrm{B}$ ". Both syringes $\mathrm{A}$ and $B$ were then simultaneously added to the reaction mixture over a 7 hour period by syringe pump. Note: it is critical that both syringes are adequately primed with the appropriate solutions to ensure no air bubbles. After addition completion, solvent was removed in vacuo and the resulting crude mixture could be purified by either Purification Methods 1 or 2 .

\section{General Procedure 6 - Stepwise Chlorinations}

To a room temperature stirred solution of triphenylphosphine oxide $(278 \mathrm{mg}, 1.0 \mathrm{mmol})$ in DMC (10 $\mathrm{mL}$ ) was added oxalyl chloride ( $86 \mu \mathrm{L}, 1.0 \mathrm{mmol}$ ) Caution: exotherm and gas evolution. The reaction mixture was stirred for 15 minutes. The appropriate alcohol $(1.0 \mathrm{mmol})$ was then added dropwise to the reaction mixture over a thirty minute period. After the addition was complete the reaction was stirred for an additional 10 minutes. CPS was regenerated by the addition of oxalyl chloride ( $86 \mu \mathrm{L}, 1.0$ $\mathrm{mmol}$ ) and the reaction was stirred for 30 minutes. The appropriate alcohol $(1.0 \mathrm{mmol})$ was again added dropwise to the reaction mixture over a thirty minute period followed by a ten minute period of stirring. A total of 5 regeneration-addition cycles were carried out in this manner.

\section{Purification Method $1-(\mathrm{COCl})_{2}$ precipitation of $\mathrm{PPh}_{3} \mathrm{O}$}

Volatiles were removed from a reaction mixture and the resulting crude was suspended in cyclohexane $(25 \mathrm{~mL}) .1 .0$ equivalent of $(\mathrm{COCl})_{2}$ (relative to the alcohol)was then added dropwise to the mixture and stirred until gas evolution had ceased. CPS was observed precipitating from solution as a white solid and the mixture was allowed to stand under an $N_{2}$ atmosphere for 1 hour. The cyclohexane layer was then isolated by filtration and solvent removed in vacuo to give the desired 
alkyl halide. Samples that had incomplete $\mathrm{PPh}_{3} \mathrm{O}$ removal (detected by ${ }^{1} \mathrm{H}-\mathrm{NMR}$ ) were treated again and allowed to stand overnight at $4{ }^{\circ} \mathrm{C}$ ensuring full removal of $\mathrm{PPh}_{3} \mathrm{O} .{ }^{1}$

\section{Purification Method 2 - Vacuum Distillation}

Solvent was removed in vacuo and the resulting crude material was purified by short path vacuum distillation to give the desired alkyl halide.

\section{Purification Method 3 - Aqueous Workup}

Reaction mixtures containing $\mathrm{LiBr}$ were first filtered to remove suspended solids. DMC was diluted with 5 volumes of water and the product was extracted with $3 \times 1$ volumes of EtOAc. EtOAc layers were combined and back extracted with $1 \times 1$ volume of water to ensure complete DMC removal. The EtOAc layer was dried over $\mathrm{MgSO}_{4}$, filtered and solvent removed in vacuo to give a crude mixture of alkyl halide and $\mathrm{PPh}_{3} \mathrm{O}$. $\mathrm{PPh}_{3} \mathrm{O}$ was removed as per Purification Method 1.

\section{Pyridyl catalyst optimisation trials}

Both a stoichiometric and catalytic chlorination reaction were investigated using pyridyl phosphine as catalyst with the following solvents: ethyl acetate, DMC, and chloroform (for comparative purposes). When ethyl acetate was employed as reaction solvent, no CPS was observed forming by ${ }^{31}$ P-NMR. Instead a species was observed slowly forming at $60 \mathrm{ppm}$, presumably a pentavalent $\mathrm{P}(\mathrm{V})$ species of the type reported by Gilheany et al. ${ }^{2}$ When DMC was utilised as solvent no conversion to product was observed ( $100 \%$ conversion to oxalates). Pre-mixing 1.0 equivalents of Hünig's base with the benzyl alcohol showed a significant improvement to 55\% chloride. Adjusting this amount down from 1.0 equivalents to 0.2 equivalents gave a poor result of $100 \%$ oxalate side product and adjusting up to 2.0 equivalents again gave $100 \%$ oxalate. Disappointingly, the pyridyl catalyst system only gave $4 \%$ of the desired chloride product when chloroform was used as solvent and thus no further work was carried out using this system.

\begin{tabular}{|c|c|c|c|}
\hline Entry & Solvent & Equiv. Hünig's base & c\% Conversion \\
\hline 1 & $\mathrm{CHCl}_{3}$ & N/A & $4 \% \mathrm{Cl}$ \\
& & & $96 \% \mathrm{Ox}$ \\
\hline 2 & EtOAc & N/A & $\mathrm{P}(\mathrm{V})$ species forms \\
\hline 3 & EtOAc & 0.2 & $0 \% \mathrm{Cl}$ \\
& & & $>99 \% \mathrm{Ox}$ \\
\hline 4 & DMC & N/A & $0 \% \mathrm{Cl}$ \\
& & & $>99 \% \mathrm{Ox}$ \\
\hline
\end{tabular}




\begin{tabular}{|c|c|c|c|}
\hline 5 & DMC & 0.2 & $0 \% \mathrm{Cl}$ \\
& & & $>99 \% \mathrm{Ox}$ \\
\hline 6 & DMC & 0.6 & $25 \% \mathrm{Cl}$ \\
& & & $75 \% \mathrm{Ox}$ \\
\hline 7 & DMC & 1.0 & $55 \% \mathrm{Cl}$ \\
& & & $45 \%$ Ox \\
\hline 8 & DMC & 2.0 & $0 \% \mathrm{Cl}$ \\
& & & $>99 \%$ Ox \\
\hline 9 & DMC & 1.0 premixed with catalyst & $>99 \%$ Ox \\
\hline
\end{tabular}

Table S1: Appel reaction solvent screen for pyridyl catalyst. Catalyst loading $15 \mathrm{~mol} \% \mathrm{PPy}(\mathrm{Ph})_{2} \mathrm{O}, 5 \mathrm{~mL}$ solvent, $1 \mathrm{mmol} \mathrm{BnOH}$, $1.05 \mathrm{mmol}(\mathrm{COCl})_{2 .} . \%$ Conversion to product colour coded according to a traffic light system; green $=75-100 \%$, amber $=26$ $74 \%$, red $=0-25 \%$.

\section{Compound Characterisation}

\section{Alkyl Chlorides}<smiles>ClCc1ccccc1</smiles>

Benzylchloride 4

Benzylchloride was synthesised according to general procedure 3 using benzyl alcohol (108 mg, 1.0 mmol). HPLC analysis showed $83 \%$ conversion to chloride product. The crude reaction mixture was purified by purification method 1 to give the target compound as a pale yellow oil. A small portion was also isolated by vacuum distillation for NMR comparison purposes.

Yield: 83\% (105 mg, $0.83 \mathrm{mmol})$

$\delta_{H}(400 \mathrm{MHz}$, Chloroform-d) $7.48-7.34(5 \mathrm{H}, \mathrm{m}), 4.64(2 \mathrm{H}, \mathrm{s})$.

$\delta_{c}(101 \mathrm{MHz}$, Chloroform-d) 137.5, 128.8, 128.6, 128.4, 46.3.

${ }^{1} \mathrm{H}-\mathrm{NMR}$ and ${ }^{13} \mathrm{C}-\mathrm{NMR}$ were in agreement with the literature. ${ }^{3}$ 

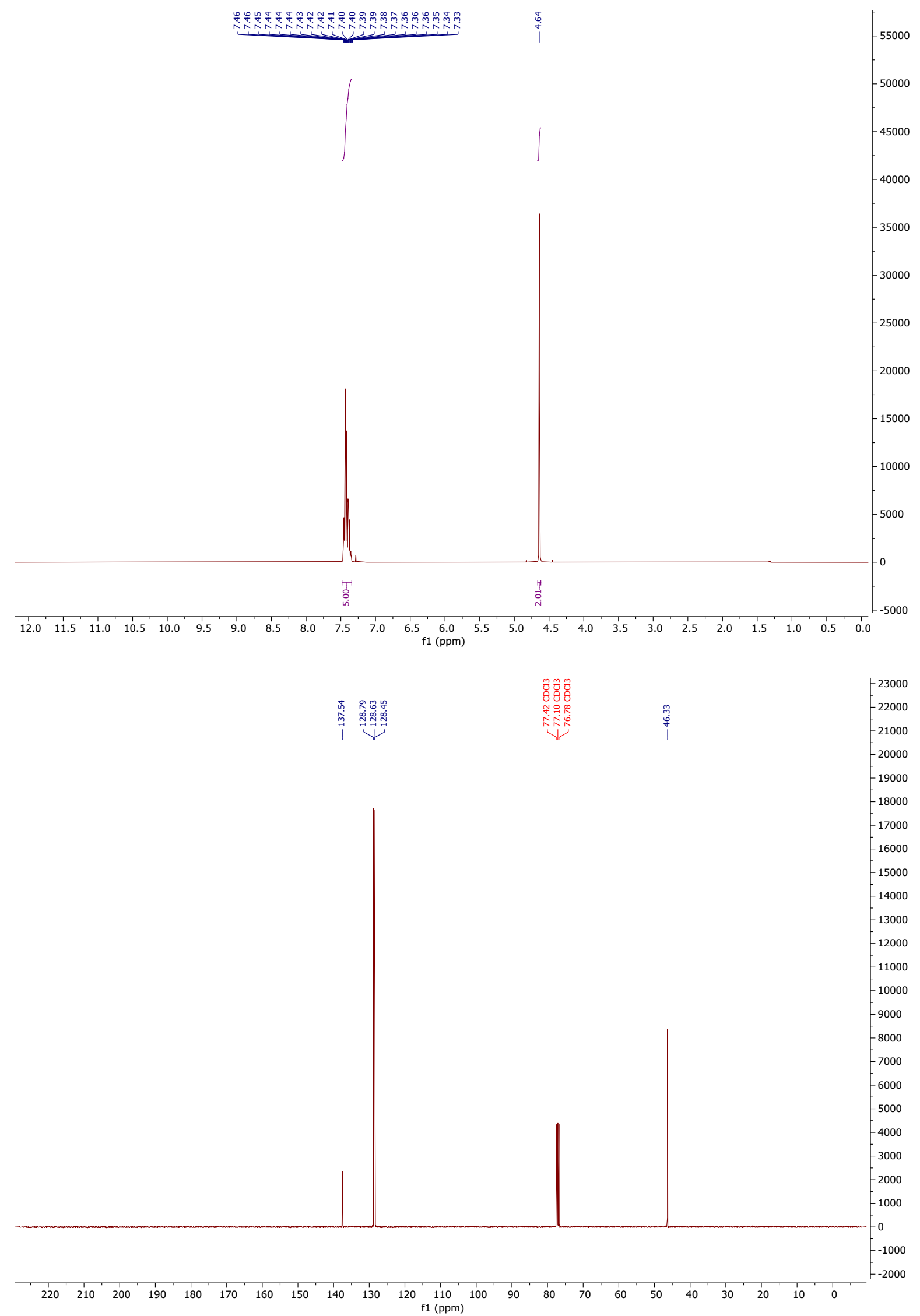
$\overbrace{}^{C l}$

(2-chloroethyl)benzene 8

(2-chloroethyl)benzene was synthesised according to general procedure 3 using phenyl-2-ethanol (122 mg, $1.0 \mathrm{mmol})$. HPLC analysis of the crude reaction mixture showed $92 \%$ conversion to product. The crude reaction mixture was purified by purification method 1 to give the target compound as a pale yellow oil, 92\% pure by HPLC. The product was further purified by short path vacuum distillation to remove the dioxalate impurities to give the target compound as a colourless oil. Yield: $66 \%$ (93 mg, $0.66 \mathrm{mmol})$.

(2-chloroethyl)benzene was also synthesised according to catalytic procedure 3 using phenyl-2ethanol (1.22 g, $10.0 \mathrm{mmol})$. The crude reaction mixture was purified according to purification method 2 to give the target compound as a colourless oil. Yield: $82 \%$ yield ( $1.15 \mathrm{~g}, 8.18 \mathrm{mmol}$ ).

Chemical Formula: $\mathrm{C}_{8} \mathrm{H}_{9} \mathrm{Cl}$

Molecular Weight: $140.61 \mathrm{gmol}^{-1}$

$\delta_{H}(400 \mathrm{MHz}$, Chloroform-d) $7.41-7.33(2 \mathrm{H}, \mathrm{m}), 7.33-7.23(3 \mathrm{H}, \mathrm{m}), 3.76(2 \mathrm{H}, \mathrm{t}, \mathrm{J}$ 7.5), $3.11(2 \mathrm{H}, \mathrm{t}$, J 7.5).

$\delta_{c}(101 \mathrm{MHz}$, Chloroform-d) 138.1, 128.9, 128.6, 126.9, 45.0, 39.2.

${ }^{1} \mathrm{H}-\mathrm{NMR}$ and ${ }^{13} \mathrm{C}-\mathrm{NMR}$ were in agreement with the literature. ${ }^{4}$ 

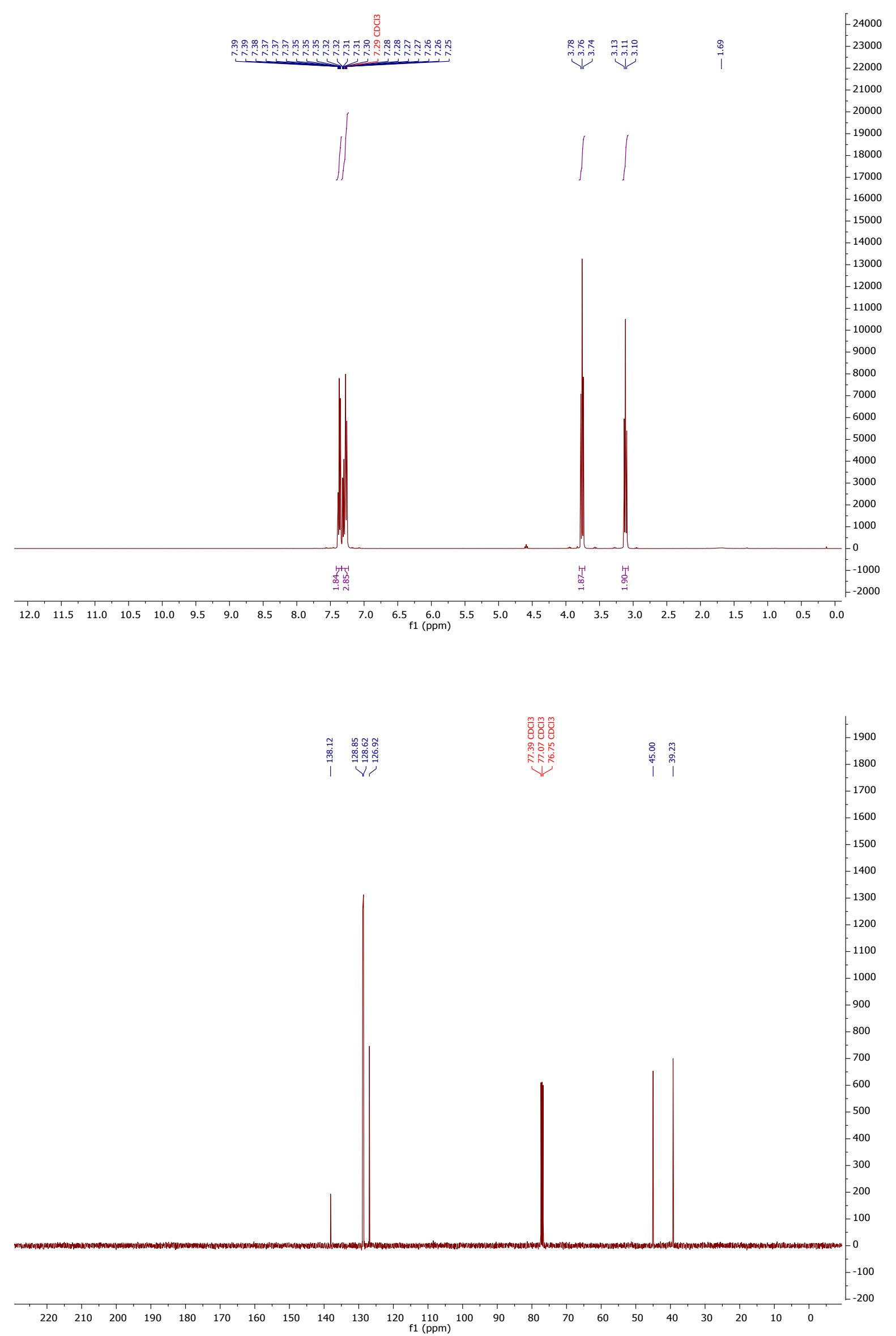
<smiles>CCCCCCCCCCCl</smiles>

Decyl chloride 9

Decyl chloride was synthesised according to general procedure 3 using decan-1-ol (158 mg, $1.0 \mathrm{mmol}$ ). ${ }^{1} \mathrm{H}$-NMR of the crude mixture showed $90 \%$ conversion to chloride product. The reaction mixture was purified by purification method 2 to give the target compounds as a colourless oil.

Chemical Formula: $\mathrm{C}_{10} \mathrm{H}_{21} \mathrm{Cl}$

Molecular Weight: $176.73 \mathrm{gmol}^{-1}$

Yield: 71\% yield (124 mg, $0.71 \mathrm{mmol})$

$\delta_{\text {н }}(500 \mathrm{MHz}$, Chloroform-d) $3.53(2 \mathrm{H}, \mathrm{t}, \mathrm{J} 6.8), 1.76(2 \mathrm{H}, \mathrm{dt}, J \mathrm{l}$. $4.8,6.8), 1.48-1.38(2 \mathrm{H}, \mathrm{m}), 1.33-$ $1.20(12 \mathrm{H}, \mathrm{m}), 0.88(3 \mathrm{H}, \mathrm{t}, \mathrm{J} 6.9)$.

$\delta_{C}(126 \mathrm{MHz}$, Chloroform-d) 45.2, 32.7, 31.9, 29.5, 29.50, 29.3, 28.9, 26.9, 22.7, 14.1.

${ }^{1} \mathrm{H}-\mathrm{NMR}$ and ${ }^{13} \mathrm{C}-\mathrm{NMR}$ were in agreement with the literature. ${ }^{3}$ 

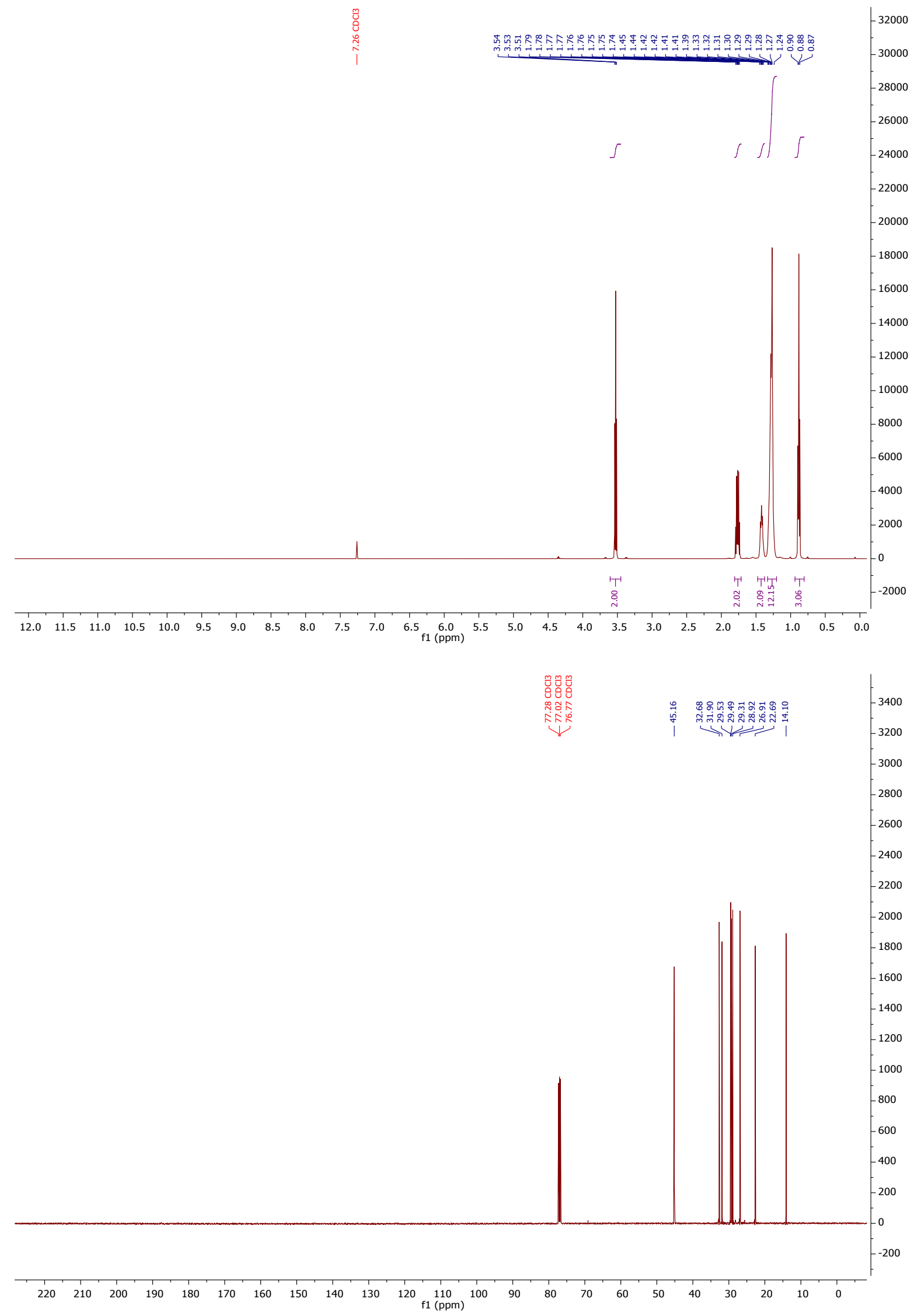


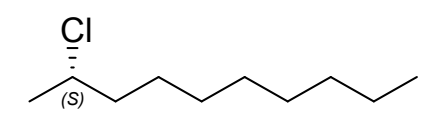

(S)-2-chlorodecane 10

(S)-2-chlorodecane was synthesised according to general procedure 3 using $(R)$-decan-2-ol (158 mg, $1.0 \mathrm{mmol}$ ). ${ }^{1} \mathrm{H}-\mathrm{NMR}$ of the crude mixture showed quantitative conversion to chloride product. The crude reaction mixture was purified by purification method 1 to give the target compound as a pale yellow oil.

Chemical Formula: $\mathrm{C}_{10} \mathrm{H}_{21} \mathrm{Cl}$

Molecular Weight: $176.73 \mathrm{gmol}^{-1}$

Yield: 62\%, (110 mg, $0.62 \mathrm{mmol})$.

$[\alpha]_{D}^{20}=+32.0\left(1.0 \mathrm{c}, \mathrm{CHCl}_{3}\right)$, literature: $[\alpha]_{\mathrm{D}}{ }^{25}+33.0\left(0.97 \mathrm{c}, \mathrm{CH}_{2} \mathrm{Cl}_{2}\right) .^{5}$

(R)-decan-2-ol: $[\alpha]_{D}^{20}=-8.0\left(1.0 \mathrm{c}, \mathrm{CHCl}_{3}\right)$, literature: $[\alpha]_{\mathrm{D}}^{23}-8.0\left(0.6 \mathrm{c} . \mathrm{CHCl}_{3}\right) .^{6}$

$\delta_{H}(500 \mathrm{MHz}$, Chloroform-d) $4.14-3.94(1 \mathrm{H}, \mathrm{m}), 1.78-1.68(2 \mathrm{H}, \mathrm{m}), 1.52(3 \mathrm{H}, \mathrm{d}, J \mathrm{~J} .6), 1.43-1.25$ $(12 \mathrm{H}, \mathrm{m}), 0.91(3 \mathrm{H}, \mathrm{t}, \mathrm{J} 6.9)$.

$\delta_{C}(126 \mathrm{MHz}$, Chloroform-d) 58.9, 40.4, 31.9, 29.5, 29.3, 29.2, 26.7, 25.4, 22.7, 14.1. 

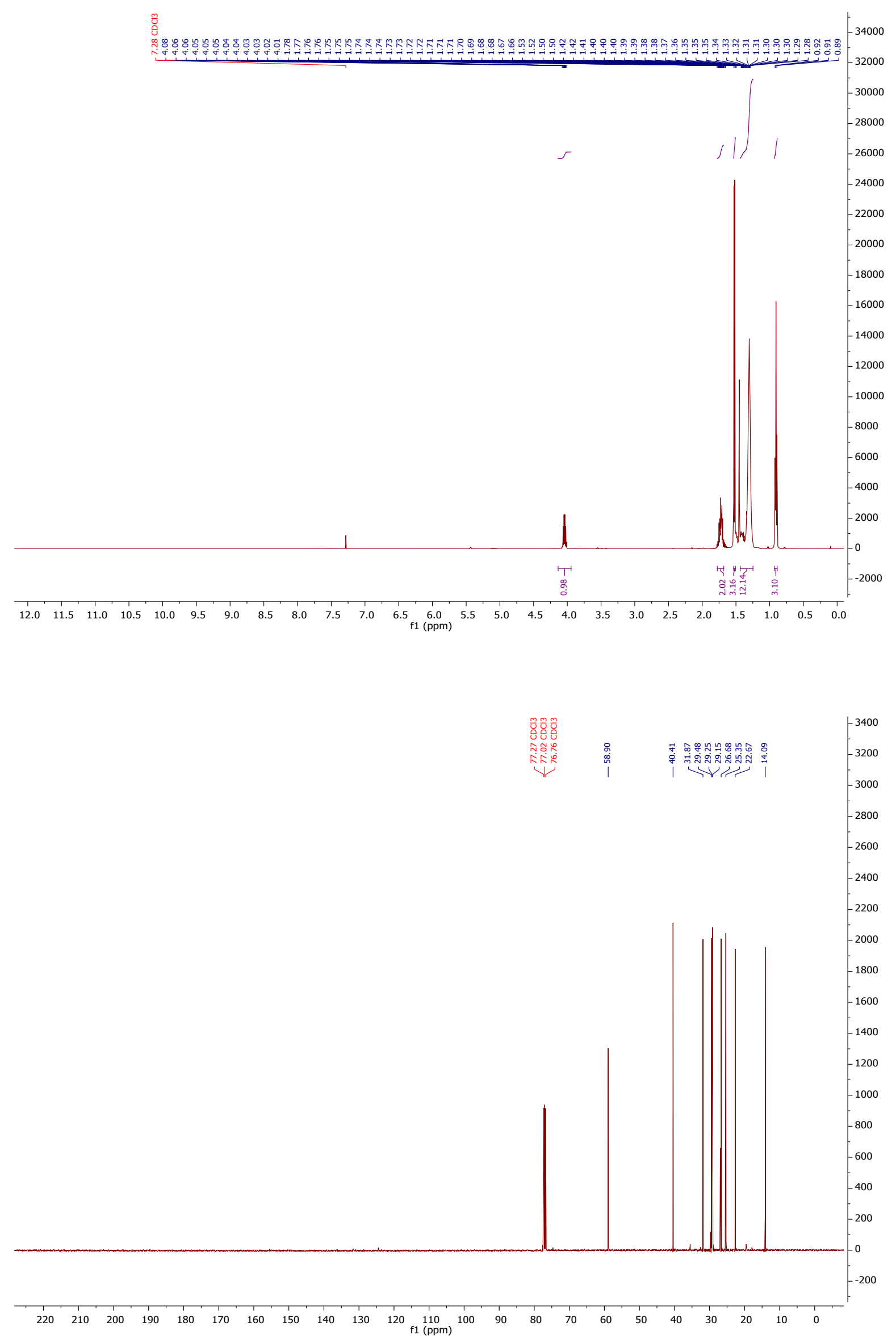


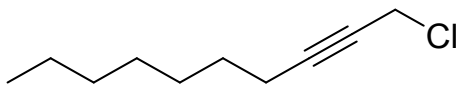

1-chlorodec-2-yne 11

1-chlorodec-2-yne was synthesised according to general procedure 3 using dec-2-yn-1-ol (154 mg, $1.00 \mathrm{mmol}$ ). ${ }^{1} \mathrm{H}-\mathrm{NMR}$ analysis of the crude reaction mixture showed $98 \%$ conversion to product. The crude reaction mixture was purified by purification method 1 to give the target compound as a pale yellow oil.

Chemical Formula: $\mathrm{C}_{10} \mathrm{H}_{17} \mathrm{Cl}$

Molecular Weight: $172.70 \mathrm{gmol}^{-1}$

Yield: 81\% (139 mg, $0.81 \mathrm{mmol})$

$\delta_{\mathrm{H}}(500 \mathrm{MHz}$, Chloroform-d) $4.14(2 \mathrm{H}, \mathrm{t}, \mathrm{J} 2.5), 2.22(2 \mathrm{H}, \mathrm{tt}, \mathrm{J}$ 7.1, 2.5), $1.54-1.48(2 \mathrm{H}, \mathrm{m}), 1.38-$ $1.25(8 \mathrm{H}, \mathrm{m}), 0.88(3 \mathrm{H}, \mathrm{t}, \mathrm{J} 6.8)$.

$\delta_{c}(126 \mathrm{MHz}$, Chloroform-d) 87.9, 75.0, 31.9, 31.5, 28.9, 28.9, 28.5, 22.8, 18.9, 14.2.

${ }^{1} \mathrm{H}-\mathrm{NMR}$ and ${ }^{13} \mathrm{C}$-NMR were in agreement with the literature. ${ }^{3}$ 

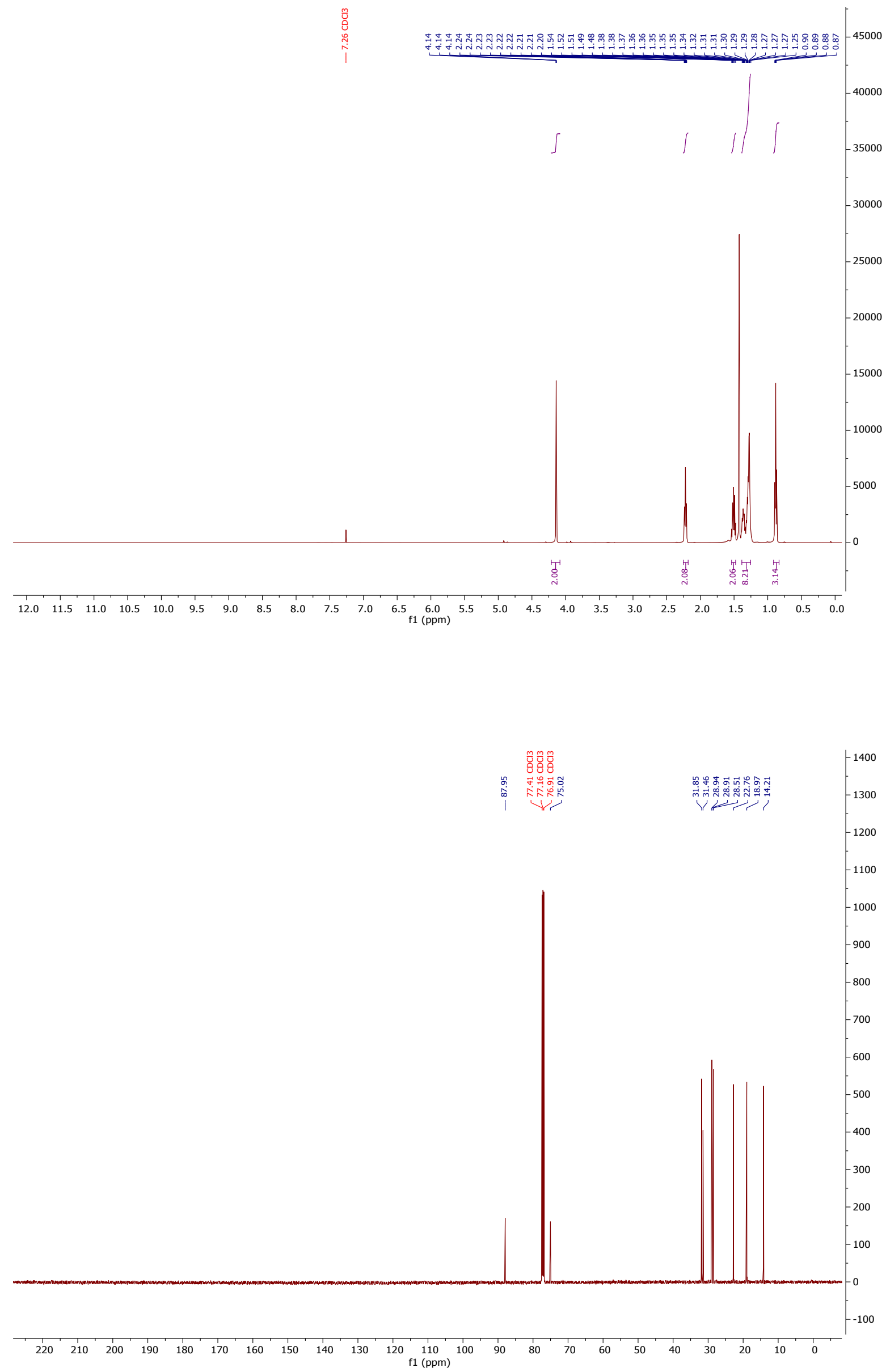
$\curvearrowright \mathrm{Cl}$

Cinnamyl chloride 12

Cinnamyl chloride was synthesised according to general procedure 3 using cinnamyl alcohol $(134 \mathrm{mg}$, $1.00 \mathrm{mmol})$. HPLC analysis of the crude reaction mixture showed $63 \%$ conversion to product. The crude reaction mixture was purified by purification method 2 to give the target compound as a colourless oil.

Chemical Formula: $\mathrm{C}_{9} \mathrm{H}_{9} \mathrm{Cl}$

Molecular Weight: $152.62 \mathrm{gmol}^{-1}$

Yield: $31 \%$ (48 $\mathrm{mg}, 0.31 \mathrm{mmol}$ )

$\delta_{H}(400 \mathrm{MHz}$, Chloroform-d) $7.48-7.40(2 \mathrm{H}, \mathrm{m}), 7.40-7.33(2 \mathrm{H}, \mathrm{m}), 7.33-7.23(1 \mathrm{H}, \mathrm{m}), 6.69(1 \mathrm{H}$, $\mathrm{d}, J$ 15.6), $6.35(1 \mathrm{H}, \mathrm{dt}, J 15.6,7.2), 4.28(2 \mathrm{H}, \mathrm{dd}, J$ 7.2, 1.2).

$\delta_{C}(101 \mathrm{MHz}$, Chloroform-d) 135.9, 134.2, 128.7, 128.3, 126.7, 124.9, 45.5.

${ }^{1} \mathrm{H}-\mathrm{NMR}$ and ${ }^{13} \mathrm{C}-\mathrm{NMR}$ were in agreement with the literature. ${ }^{3}$ 

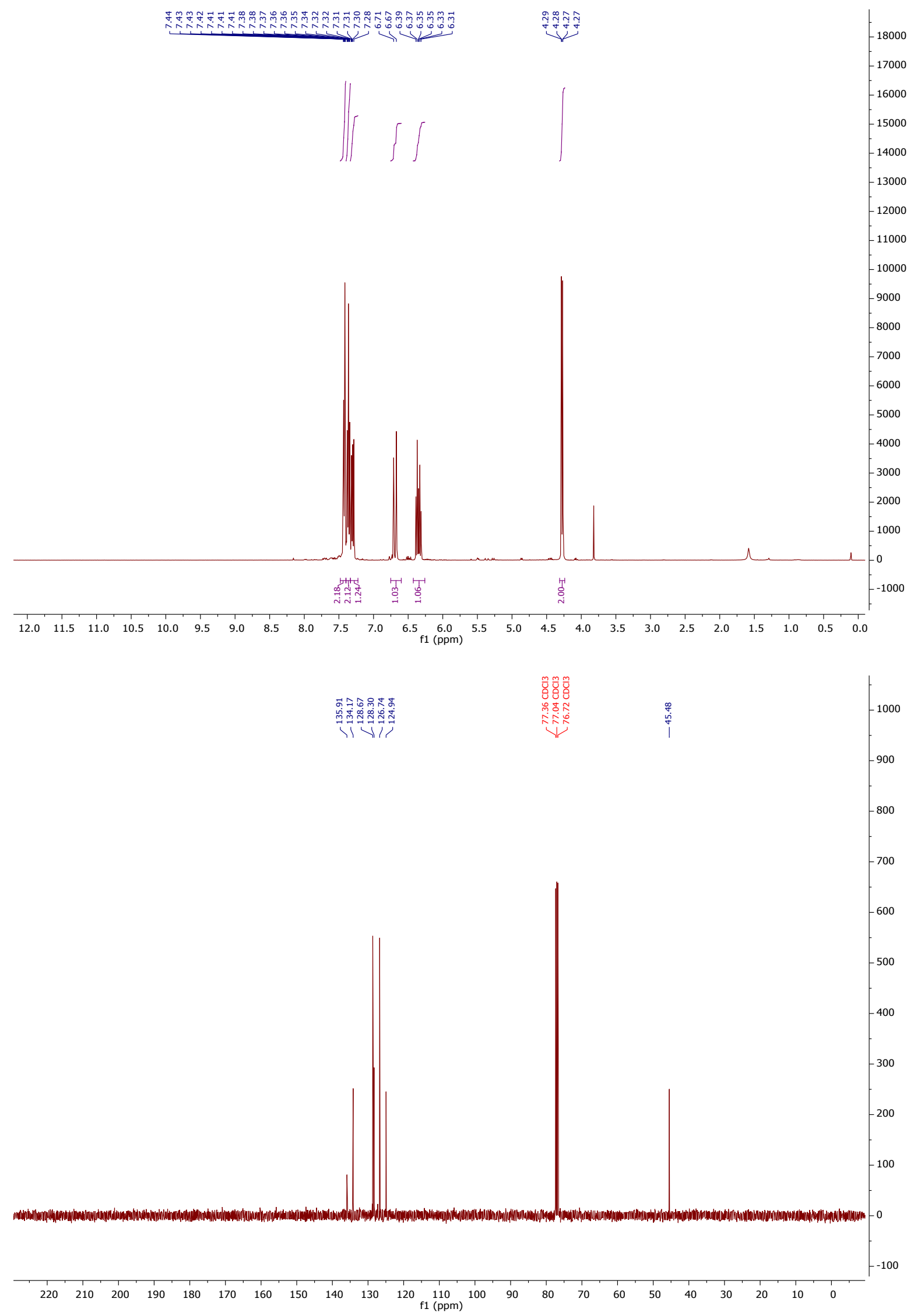
<smiles>ClC(c1ccccc1)c1ccccc1</smiles>

(Chloromethylene)dibenzene 13

(Chloromethylene)dibenzene was synthesised according to the general procedure 3 using diphenylmethanol (184 mg, $1.0 \mathrm{mmol}$ ). HPLC analysis showed >99\% conversion to chloride product. The crude reaction mixture was purified by purification method 1 to give the target compound as pale yellow oil.

Chemical Formula: $\mathrm{C}_{13} \mathrm{H}_{11} \mathrm{Cl}$

Molecular Weight: $202.68 \mathrm{gmol}^{-1}$

Yield: 63\% (128 mg, $0.63 \mathrm{mmol})$.

$\delta_{H}(500 \mathrm{MHz}$, Chloroform-d) $7.53-7.45(4 \mathrm{H}, \mathrm{m}), 7.44-7.39(4 \mathrm{H}, \mathrm{m}), 7.37-7.33(2 \mathrm{H}, \mathrm{m}), 6.20(1 \mathrm{H}$, s).

$\delta_{c}(126 \mathrm{MHz}$, Chloroform-d) 141.1, 128.6, 128.1, 127.8, 64.3, 26.9.

${ }^{1} \mathrm{H}-\mathrm{NMR}$ and ${ }^{13} \mathrm{C}$-NMR were in agreement with the literature. ${ }^{7}$ 

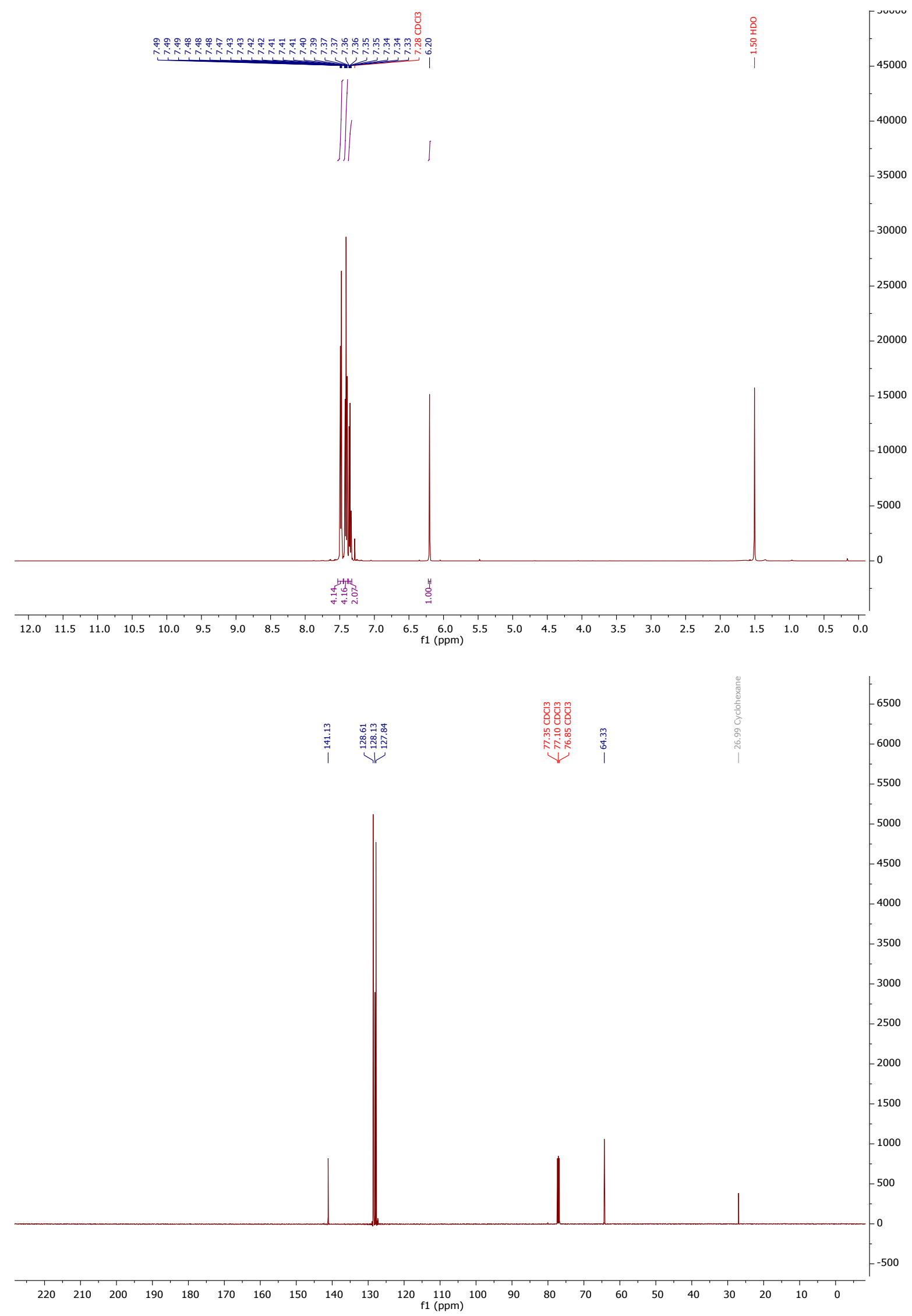
$\mathrm{Q}^{\mathrm{Cl}}$

3-chlorocyclohex-1-ene 14

3-chlorocyclohex-1-ene was synthesised according to general procedure 3 using cyclohexen-3-ol (98 $\mathrm{mg}, 1.0 \mathrm{mmol}$ ). ${ }^{1} \mathrm{H}-\mathrm{NMR}$ analysis of the crude reaction mixture showed $75 \%$ conversion to chloride product. The crude reaction mixture was purified by purification method 2 but co-distilled with DMC and was inseparable.

Chemical Formula: $\mathrm{C}_{6} \mathrm{H}_{9} \mathrm{Cl}$

Molecular Weight: $116.59 \mathrm{gmol}^{-1}$

$\delta_{\mathrm{H}}(500 \mathrm{MHz}$, Chloroform-d) $5.87-5.74(2 \mathrm{H}, \mathrm{m}), 4.58(1 \mathrm{H}, \mathrm{tq}, J \mathrm{~J} .0,3.1,2.4), 2.12-1.97(4 \mathrm{H}, \mathrm{m})$, $1.90-1.81(1 \mathrm{H}, \mathrm{m}), 1.68-1.58(1 \mathrm{H}, \mathrm{m})$.

$\delta_{c}(126 \mathrm{MHz}$, Chloroform-d) 131.2, 128.0, 54.7, 32.3, 24.6, 18.3.

${ }^{1} \mathrm{H}-\mathrm{NMR}$ and ${ }^{13} \mathrm{C}-\mathrm{NMR}$ were in agreement with the literature. ${ }^{8}$ 

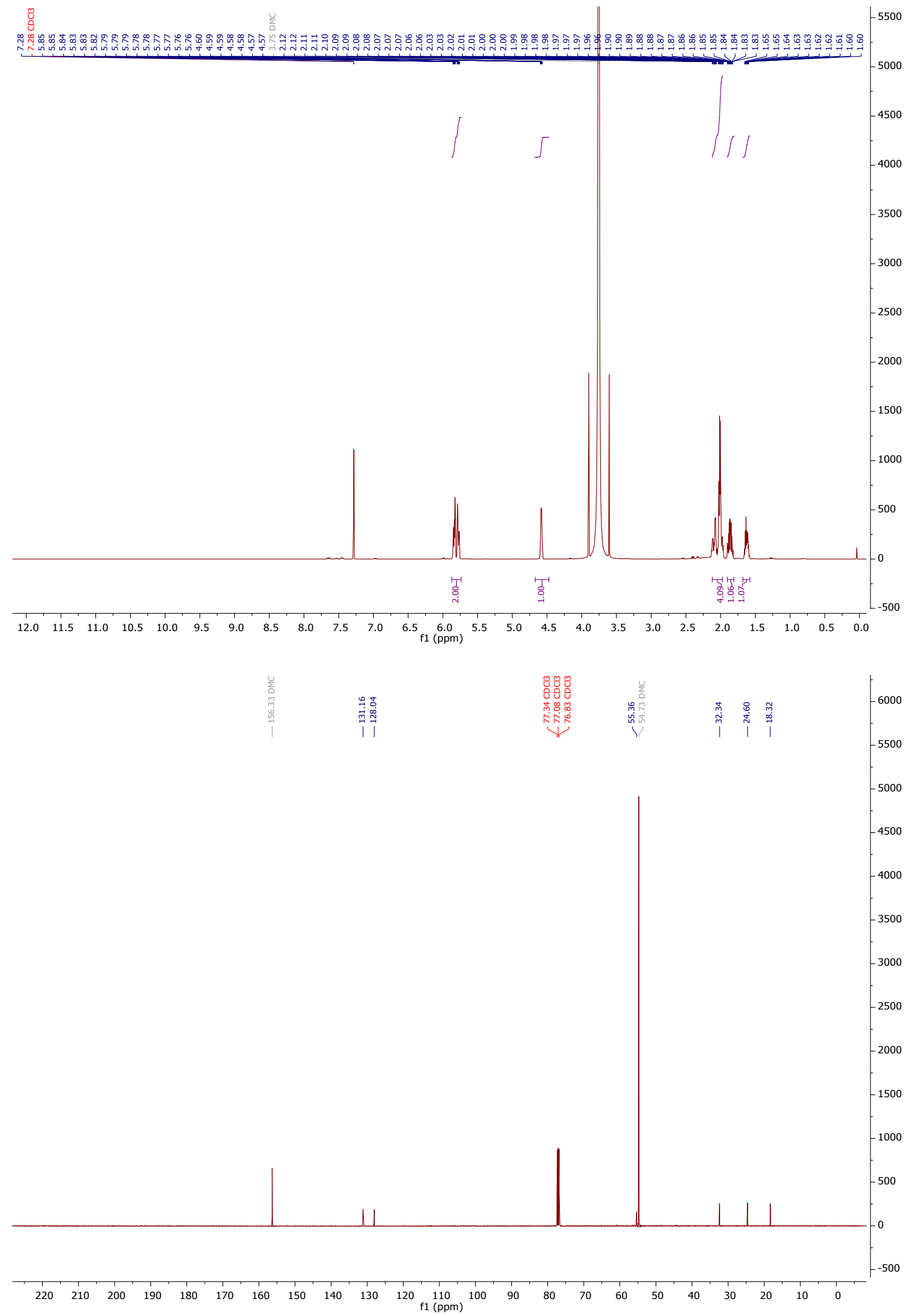
<smiles>CC(Cl)c1ccc(Br)cc1</smiles>

1-bromo-4-(1-chloroethyl)benzene 15

1-bromo-4-(1-chloroethyl)benzene was synthesised according to general procedure 3 using 1-(4bromophenyl)ethan-1-ol (100 mg, $0.5 \mathrm{mmol}$ ). HPLC analysis showed $>99 \%$ conversion to chloride product. The crude reaction mixture was purified by purification method 1 to give the target compound as pale yellow oil. Yield: $74 \%(81 \mathrm{mg}, 0.37 \mathrm{mmol})$

1-bromo-4-(1-chloroethyl)benzene was also synthesised according to catalytic procedure 3 using 1-(4bromophenyl)ethan-1-ol (1.81 g, $9.0 \mathrm{mmol})$. The crude reaction mixture was purified according to purification method 2 to give the target compound as a pale yellow oil. Yield: $81 \%$ yield $(1.60 \mathrm{~g}, 7.3$ $\mathrm{mmol})$.

1-bromo-4-(1-chloroethyl)benzene was also synthesised using traditional Appel reaction conditions. To a stirred solution of $\mathrm{PPh}_{3}(2.62 \mathrm{~g}, 10.0 \mathrm{mmol})$ in $\mathrm{DCM}(50 \mathrm{~mL})$ was added $\mathrm{CCl}_{4}(0.96 \mathrm{~mL}, 10.0 \mathrm{mmol})$. The reaction mixture was stirred for 30 minutes. 1-(4-bromophenyl)ethan-1-ol (2.01 g, $10.0 \mathrm{mmol})$ was then added to the reaction mixture and stirred overnight at room temperature. Volatiles were removed in vacuo to give a crude mixture. The product was isolated from the reaction mixture by purification method 1 to give a colourless oil. Yield: $74 \%$ (1.681 g, $7.37 \mathrm{mmol})$.

Chemical Formula: $\mathrm{C}_{8} \mathrm{H}_{8} \mathrm{BrCl}$

Molecular Weight: $219.51 \mathrm{gmol}^{-1}$

$\delta_{H}(500 \mathrm{MHz}$, Chloroform-d) 7.51- $7.46(2 \mathrm{H}, \mathrm{m}), 7.32-7.27(2 \mathrm{H}, \mathrm{m}), 5.04(1 \mathrm{H}, \mathrm{q}, \mathrm{J} 6.8), 1.83(3 \mathrm{H}, \mathrm{d}$, J6.8)

$\delta_{c}(126 \mathrm{MHz}$, Chloroform-d) 141.9, 131.9, 128.4, 122.2, 57.9, 26.6

${ }^{1} \mathrm{H}-\mathrm{NMR}$ and ${ }^{13} \mathrm{C}$-NMR were in agreement with the literature. ${ }^{9}$ 

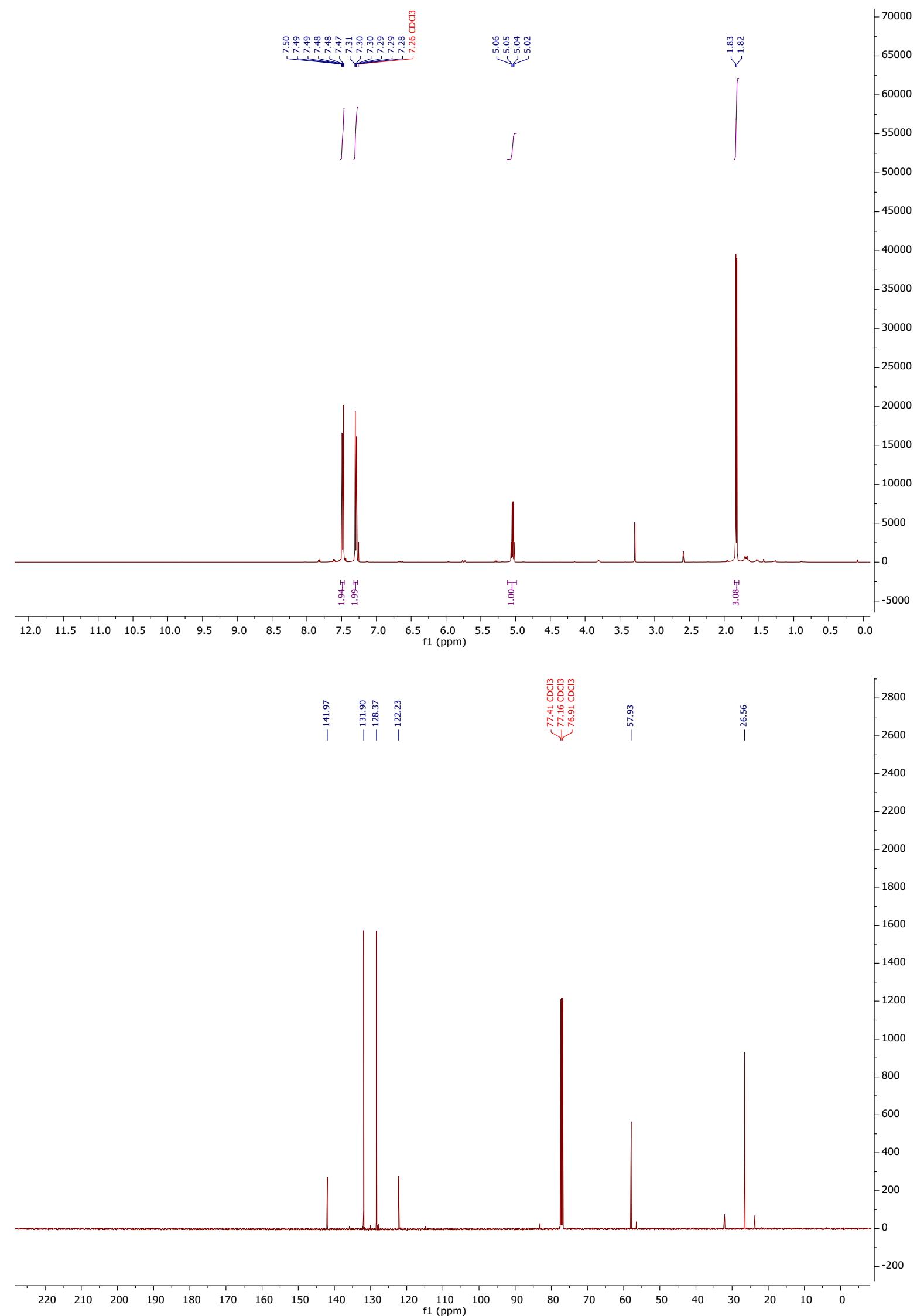
<smiles>O=C(CCl)c1ccccc1</smiles>

2-chloro-1-phenylethan-1-one 16

2-chloro-1-phenylethan-1-one was synthesised according to catalytic procedure 3 using 2-hydroxy-1phenylethan-1-one (50 mg, $0.37 \mathrm{mmol}$ ). HPLC analysis showed $92 \%$ conversion to chloride product. The crude reaction mixture was purified by purification method 2 to give the target compound as a colourless oil.

Yield: 54\% (31 mg, $0.20 \mathrm{mmol})$

Chemical Formula: $\mathrm{C}_{8} \mathrm{H}_{7} \mathrm{ClO}$

Molecular Weight: $154.59 \mathrm{gmol}^{-1}$

$\delta_{H}(500 \mathrm{MHz}$, Chloroform-d) $7.96(2 \mathrm{H}, \mathrm{dd}, \mathrm{J}$ 8.4, 1.3), $7.64-7.60(1 \mathrm{H}, \mathrm{m}), 7.53-7.46(2 \mathrm{H}, \mathrm{m}), 4.72$ $(2 \mathrm{H}, \mathrm{s})$.

$\delta_{C}(126 \mathrm{MHz}$, Chloroform-d) 191.1, 134.3, 134.0, 128.9, 128.5, 46.0.

${ }^{1} \mathrm{H}$-NMR and ${ }^{13} \mathrm{C}-\mathrm{NMR}$ were in agreement with the literature. ${ }^{10}$ 

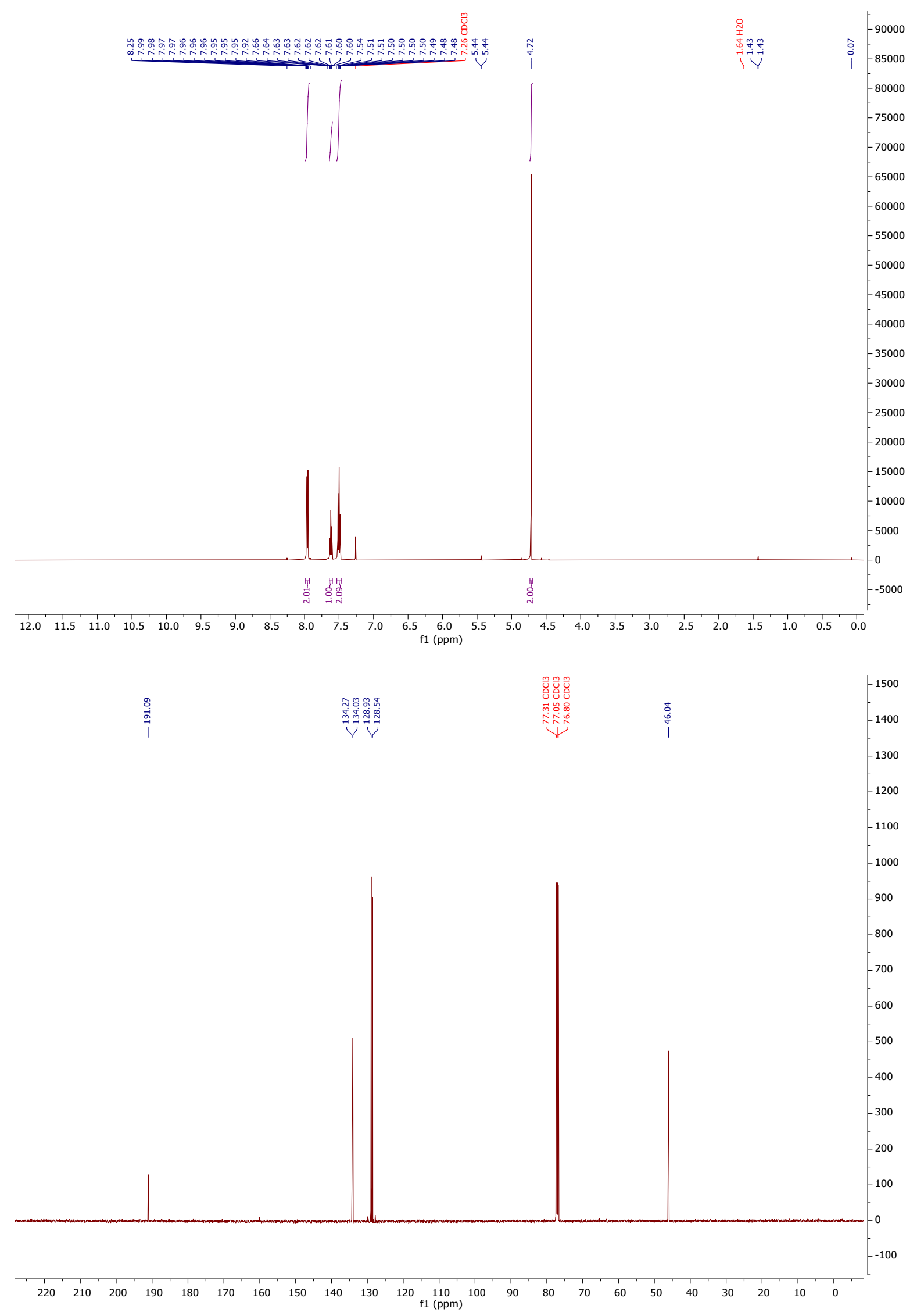
<smiles>ClCCc1c[nH]c2ccccc12</smiles>

3-(2-chloroethyl)-1H-indole 18

3-(2-chloroethyl)-1H-indole was synthesised according to catalytic procedure 3 using 2-(1H-indol-3yl)ethan-1-ol (36 mg, $0.22 \mathrm{mmol}$ ). The crude reaction mixture was purified by silica gel chromatography 0-50\% EtOAc:Heptane gradient elution to give the target compound as a colourless oil.

Molecular Weight: $179.65 \mathrm{gmol}^{-1}$

Chemical Formula: $\mathrm{C}_{10} \mathrm{H}_{10} \mathrm{ClN}$

Yield: $21 \%$ (8 $\mathrm{mg}, 0.045 \mathrm{mmol})$

$\delta_{H}(500 \mathrm{MHz}$, Chloroform-d) $8.04(1 \mathrm{H}, \mathrm{d}, J \mathrm{~J} .8), 7.41(1 \mathrm{H}, \mathrm{t}, J$ 8.2), $7.28-7.21(1 \mathrm{H}, \mathrm{m}), 7.21-7.14(1$ $H, m), 7.12(1 \mathrm{H}, \mathrm{d}, J 2.4), 3.81(2 \mathrm{H}, \mathrm{t}, J$ J 7.5$), 3.28(2 \mathrm{H}, \mathrm{t}, J \mathrm{~J} .5)$.

$\delta_{c}(126 \mathrm{MHz}$, Chloroform-d) 136.2, 127.1, 122.4, 122.3, 119.6, 118.5, 112.6, 111.3, 44.6, 29.1.

${ }^{1} \mathrm{H}-\mathrm{NMR}$ and ${ }^{13} \mathrm{C}-\mathrm{NMR}$ were in agreement with the literature. ${ }^{11}$ 


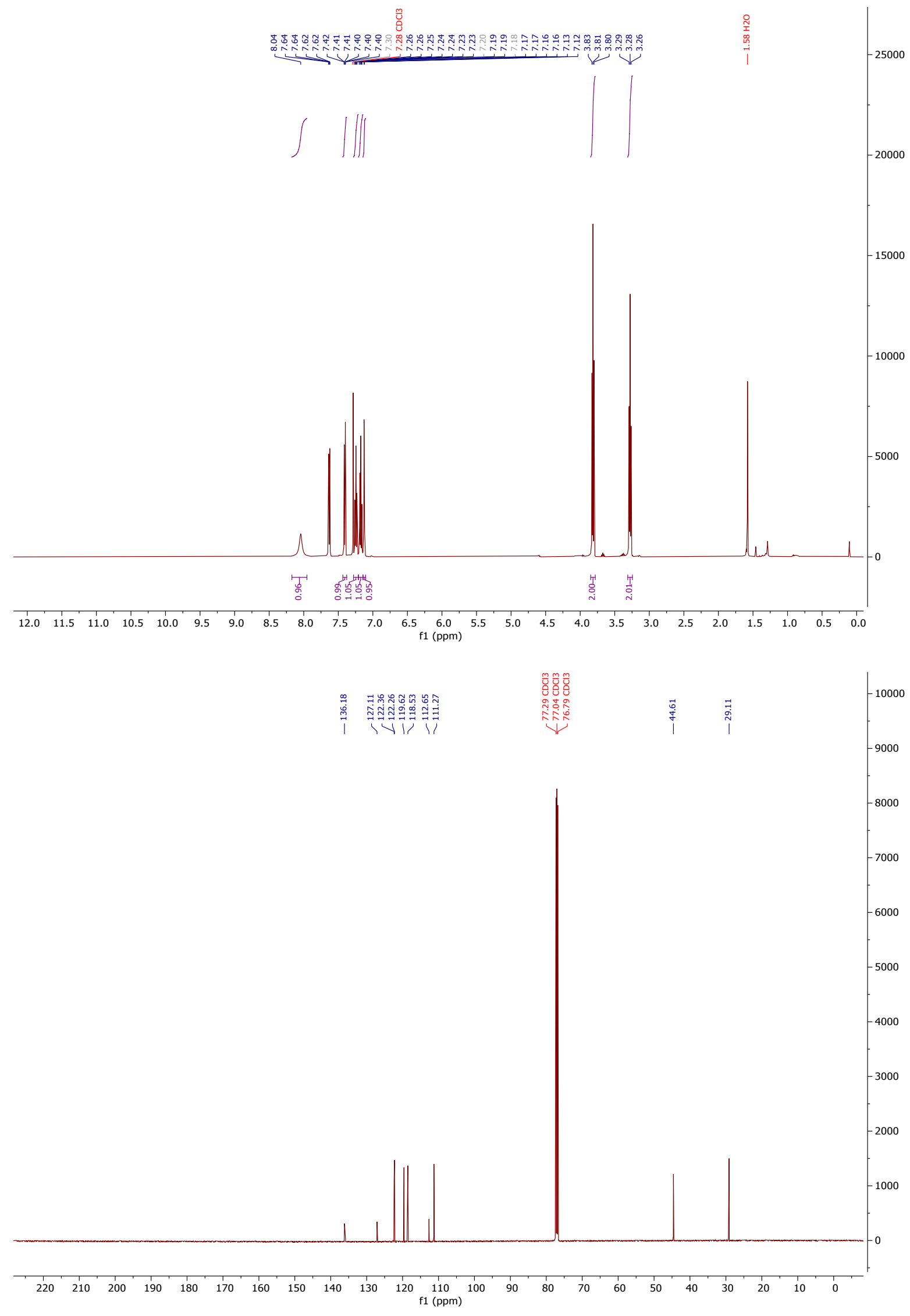




\section{Alkyl Bromides}<smiles>BrCc1ccccc1</smiles>

Benzylbromide 22

Benzylbromide was synthesised according to catalytic bromination procedure 5 using benzyl alcohol (108 mg, $1.0 \mathrm{mmol})$ and $260 \mathrm{mg} \mathrm{LiBr}(3.0 \mathrm{mmol})$. HPLC analysis showed 82\% conversion to bromide product. $\mathrm{PPh}_{3} \mathrm{O}$ was removed by purification method 1 to give the target compound as pale yellow oil. A small portion was isolated by vacuum distillation for NMR comparison purposes.

Yield: $82 \%$ (140 mg, $0.82 \mathrm{mmol}$ )

$\delta_{H}(400 \mathrm{MHz}$, Chloroform-d) $7.47-7.29(5 \mathrm{H}, \mathrm{m}), 4.54(2 \mathrm{H}, \mathrm{s})$.

$\delta_{c}(101 \mathrm{MHz}$, Chloroform-d) 137.8, 129.1, 128.8, 128.5, 33.6.

${ }^{1} \mathrm{H}-\mathrm{NMR}$ and ${ }^{13} \mathrm{C}$-NMR were in agreement with the literature. ${ }^{3}$ 

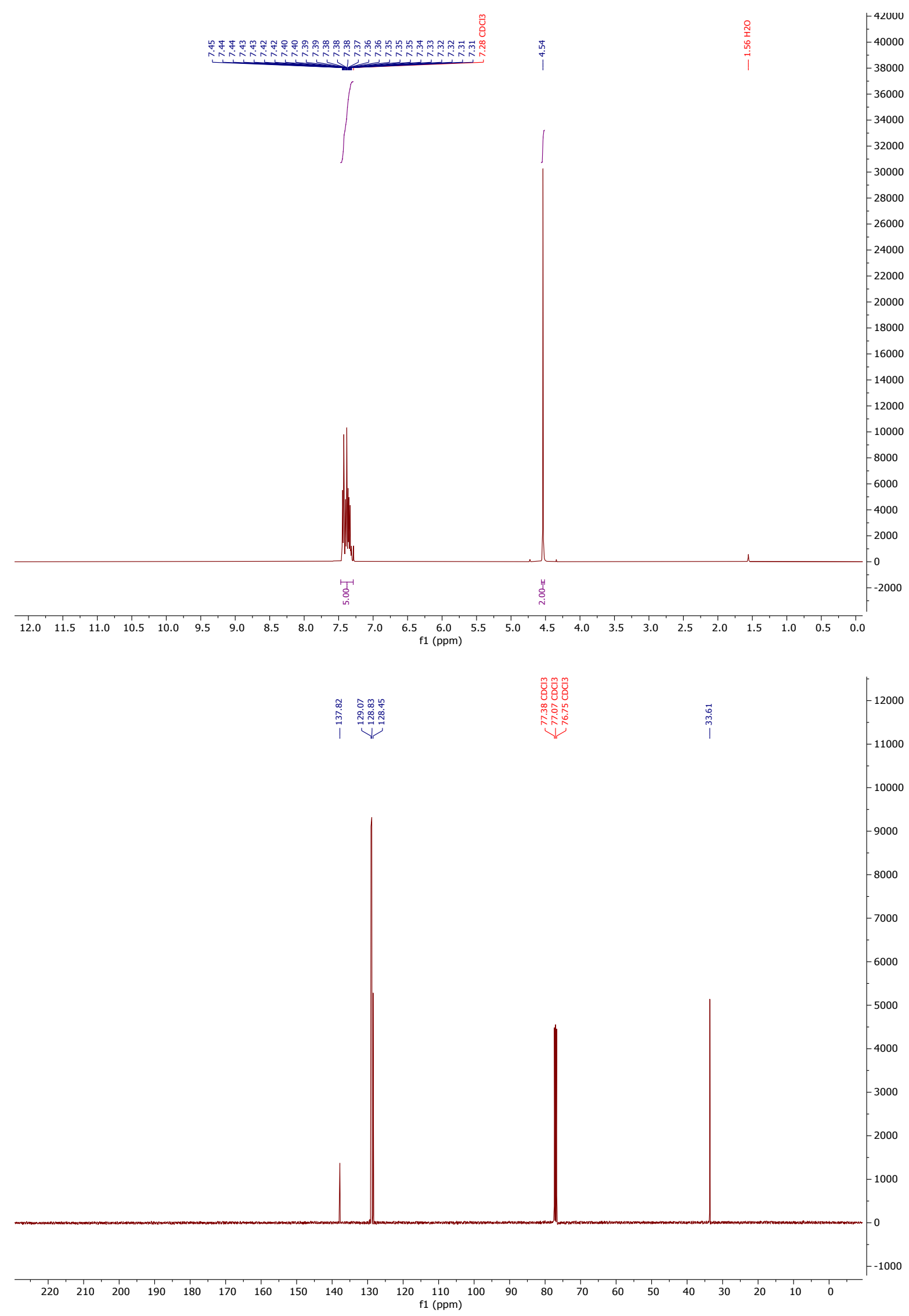
$\sim^{B r}$

(2-bromoethyl)benzene $\mathbf{2 3}$

(2-bromoethyl)benzene was synthesised according to catalytic bromination procedure 5 using 3phenylethanol (122 mg, $1.0 \mathrm{mmol}$ ) and $260 \mathrm{mg} \mathrm{LiBr}$ ( $3.0 \mathrm{mmol}$ ). HPLC analysis showed 77\% conversion to bromide product. $\mathrm{PPh}_{3} \mathrm{O}$ was removed by purification method 1 to give the target compound as pale yellow oil. The product was further purified by purification method 2 to give the target compound as a colourless oil.

Chemical Formula: $\mathrm{C}_{8} \mathrm{H}_{9} \mathrm{Br}$

Molecular Weight: $185.06 \mathrm{gmol}^{-1}$

Yield: $43 \%$ (79 mg, $0.43 \mathrm{mmol})$

$\delta_{H}(500 \mathrm{MHz}$, Chloroform-d) 7.37- $7.31(2 \mathrm{H}, \mathrm{m}), 7.29-7.20(3 \mathrm{H}, \mathrm{m}), 3.58(2 \mathrm{H}, \mathrm{t}, \mathrm{J} 7.6), 3.17(2 \mathrm{H}, \mathrm{t}$, J 7.6).

$\delta_{c}(126 \mathrm{MHz}$, Chloroform-d) 138.9, 128.7, 128.6, 126.9, 39.5, 32.9.

${ }^{1} \mathrm{H}-\mathrm{NMR}$ and ${ }^{13} \mathrm{C}-\mathrm{NMR}$ were in agreement with the literature. ${ }^{12}$ 


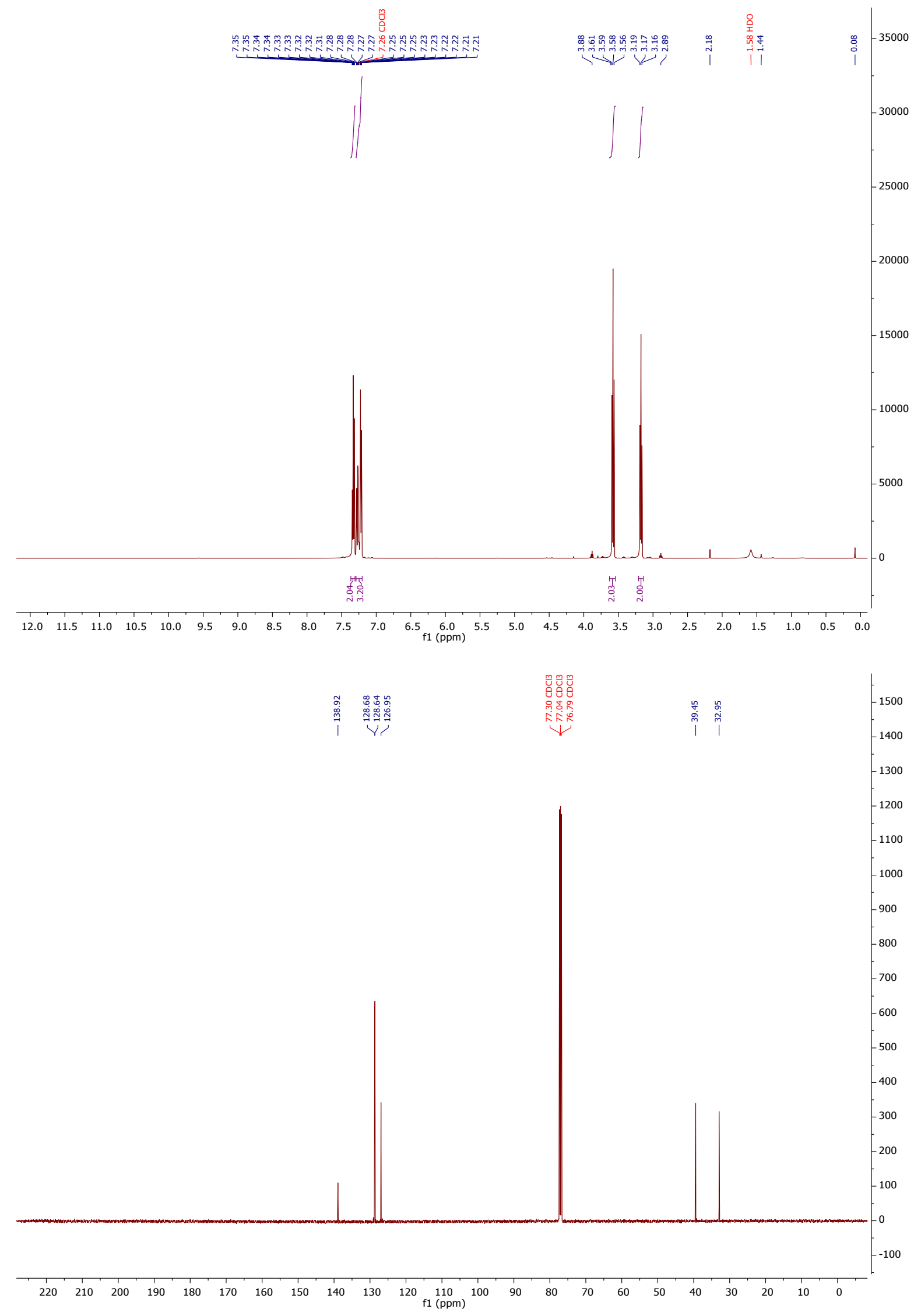


Decyl bromide $\mathbf{2 4}$

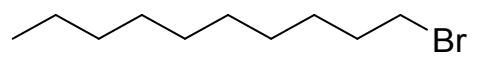

Decyl bromide was synthesised according to catalytic procedure 5 using decan-1-ol (158 mg, 1.0 mmol). ${ }^{1} \mathrm{H}-\mathrm{NMR}$ of the crude mixture showed $93 \%$ conversion to bromide product. The crude reaction mixture was purified by purification method 1 to give the target compound as a colourless oil. No further purification was conducted.

Chemical Formula: $\mathrm{C}_{10} \mathrm{H}_{21} \mathrm{Br}$

Molecular Weight: $221.18 \mathrm{gmol}^{-1}$

Yield: 65\% yield (145 mg, $0.65 \mathrm{mmol})$

$\delta_{H}(400 \mathrm{MHz}$, Chloroform-d) $3.41(2 \mathrm{H}, \mathrm{t}, J \mathrm{~J} .9), 1.91-1.80(2 \mathrm{H}, \mathrm{m}), 1.46-1.38(2 \mathrm{H}, \mathrm{m}), 1.31-1.24$ $(12 \mathrm{H}, \mathrm{m}), 0.88(3 \mathrm{H}, \mathrm{t}, \mathrm{J} 6.8)$.

$\delta_{C}(101 \mathrm{MHz}$, Chloroform-d) 34.2, 33.0, 32.0, 29.7, 29.6, 29.4, 28.9, 28.3, 22.8, 14.3.

${ }^{1} \mathrm{H}-\mathrm{NMR}$ and ${ }^{13} \mathrm{C}-\mathrm{NMR}$ were in agreement with the literature. ${ }^{3}$ 


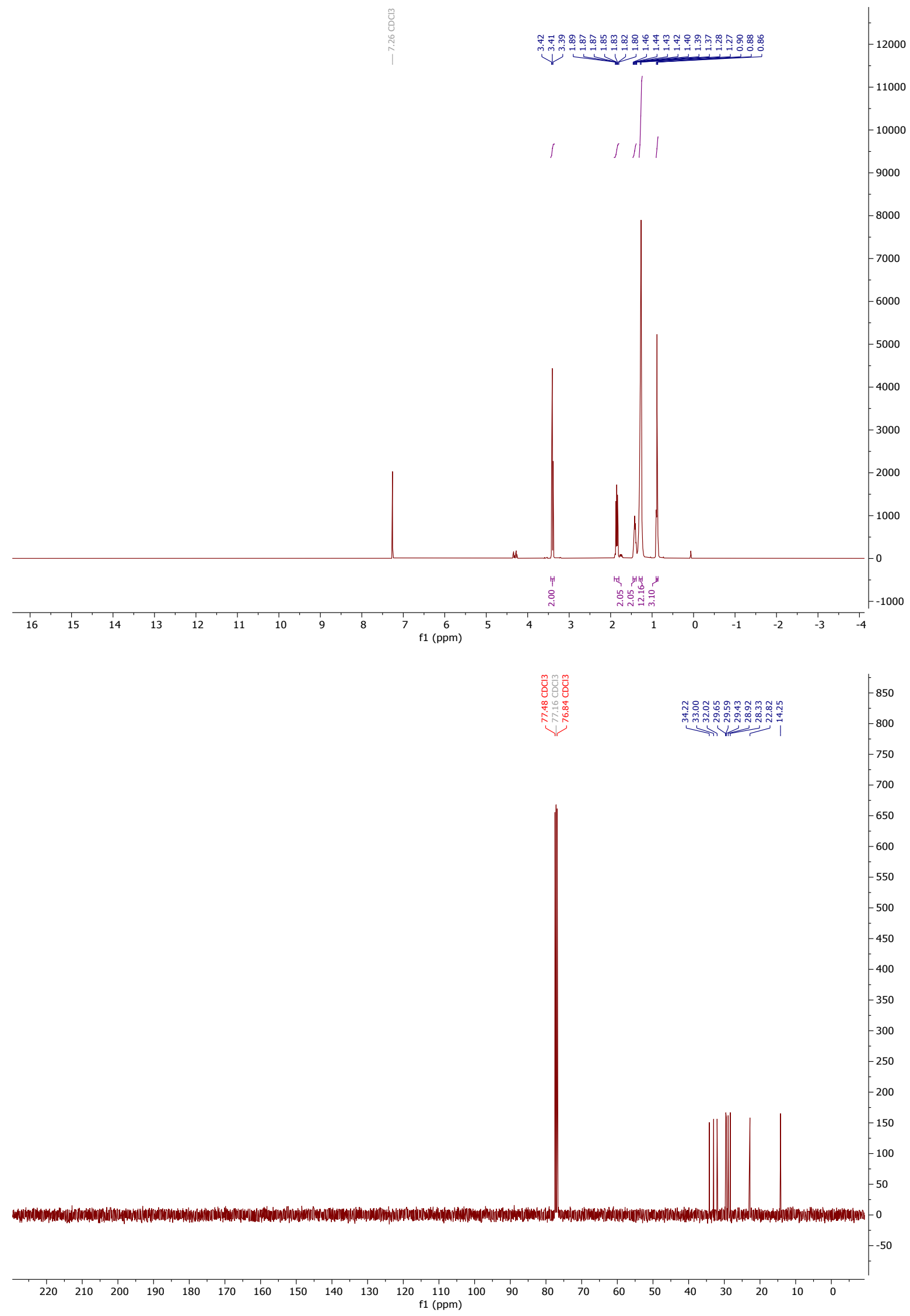




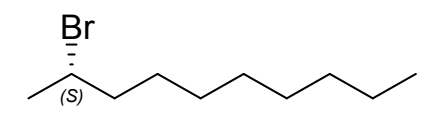

(S)-2-bromodecane 25

(S)-2-bromodecane was synthesised according to catalytic procedure 5 using $(R)$-decan-2-ol (158 $\mathrm{mg}$, $1.0 \mathrm{mmol}) .{ }^{1} \mathrm{H}-\mathrm{NMR}$ of the crude mixture showed $93 \%$ conversion to bromide product. The crude reaction mixture was purified by purification method 1 to give the target compound as a pale yellow oil.

Chemical Formula: $\mathrm{C}_{10} \mathrm{H}_{21} \mathrm{Br}$

Molecular Weight: $221.18 \mathrm{gmol}^{-1}$

Yield: 59\% yield (131 $\mathrm{mg}, 0.59 \mathrm{mmol})$

$[\alpha]_{D}^{20}=+32.0\left(1.0 \mathrm{c}, \mathrm{CHCl}_{3}\right)$, literature: $[\alpha]_{D}{ }^{25}+32.4$ (neat). ${ }^{13}$

$(R)$-decan-2-ol: $[\alpha]_{D}{ }^{20}=-8.0\left(1.0 \mathrm{c}, \mathrm{CHCl}_{3}\right)$, literature: $[\alpha]_{D}{ }^{23}-8.0\left(0.6 \mathrm{c} . \mathrm{CHCl}_{3}\right) .^{6}$

$\delta_{H}(500 \mathrm{MHz}$, Chloroform-d $) 4.13(1 \mathrm{H}, \mathrm{dqd}, \mathrm{J} 8.2,6.6,5.1), 1.89-1.65(5 \mathrm{H}, \mathrm{m}), 1.49-1.20(12 \mathrm{H}, \mathrm{m})$, $0.88(3 \mathrm{H}, \mathrm{t}, \mathrm{J} 6.9)$.

$\delta_{c}(126 \mathrm{MHz}$, Chloroform-d) 52.0, 41.2, 31.9, 29.5, 29.2, 29.0, 27.8, 26.5, 22.7, 14.1. 

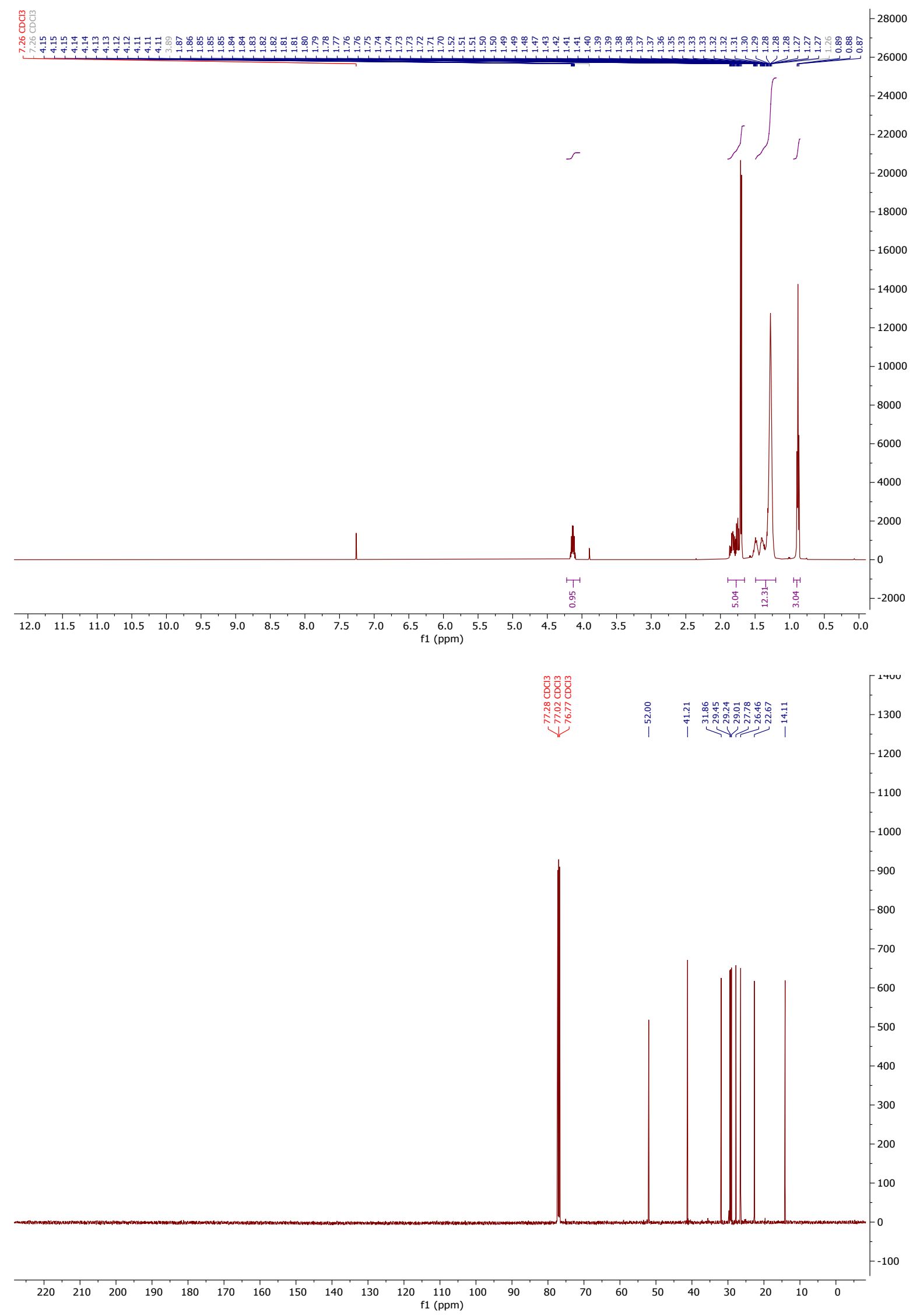


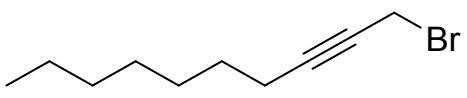

1-bromodec-2-yne 26

1-bromodec-2-yne was synthesised according to catalytic procedure 5 using dec-2-yn-1-ol (154 mg, $1.0 \mathrm{mmol}$ ). ${ }^{1} \mathrm{H}-\mathrm{NMR}$ of the crude mixture showed $94 \%$ conversion to bromide product. The crude reaction mixture was purified by purification method 1 to give the target compound as a yellow oil. No further purification was carried out.

Chemical Formula: $\mathrm{C}_{10} \mathrm{H}_{17} \mathrm{Br}$

Molecular Weight: $217.15 \mathrm{gmol}^{-1}$

Yield: 89\% (192 mg, $0.89 \mathrm{mmol})$.

$\delta_{H}(400 \mathrm{MHz}$, Chloroform-d) $3.93(2 \mathrm{H}, \mathrm{t}, \mathrm{J}$ 2.4), $2.23(2 \mathrm{H}, \mathrm{tt}, \mathrm{J}$ 7.1, 2.4), $1.51(2 \mathrm{H}, \mathrm{p}, \mathrm{J}$ 7.1), $1.36-1.26$ $(8 \mathrm{H}, \mathrm{m}), 0.88(3 \mathrm{H}, \mathrm{t})$.

$\delta_{c}(101 \mathrm{MHz}$, Chloroform-d) 88.4, 75.3, 31.7, 28.8, 28.8, 28.4, 22.6, 18.9, 15.8, 14.1.

${ }^{1} \mathrm{H}-\mathrm{NMR}$ and ${ }^{13} \mathrm{C}$-NMR were in agreement with the literature. ${ }^{3}$ 


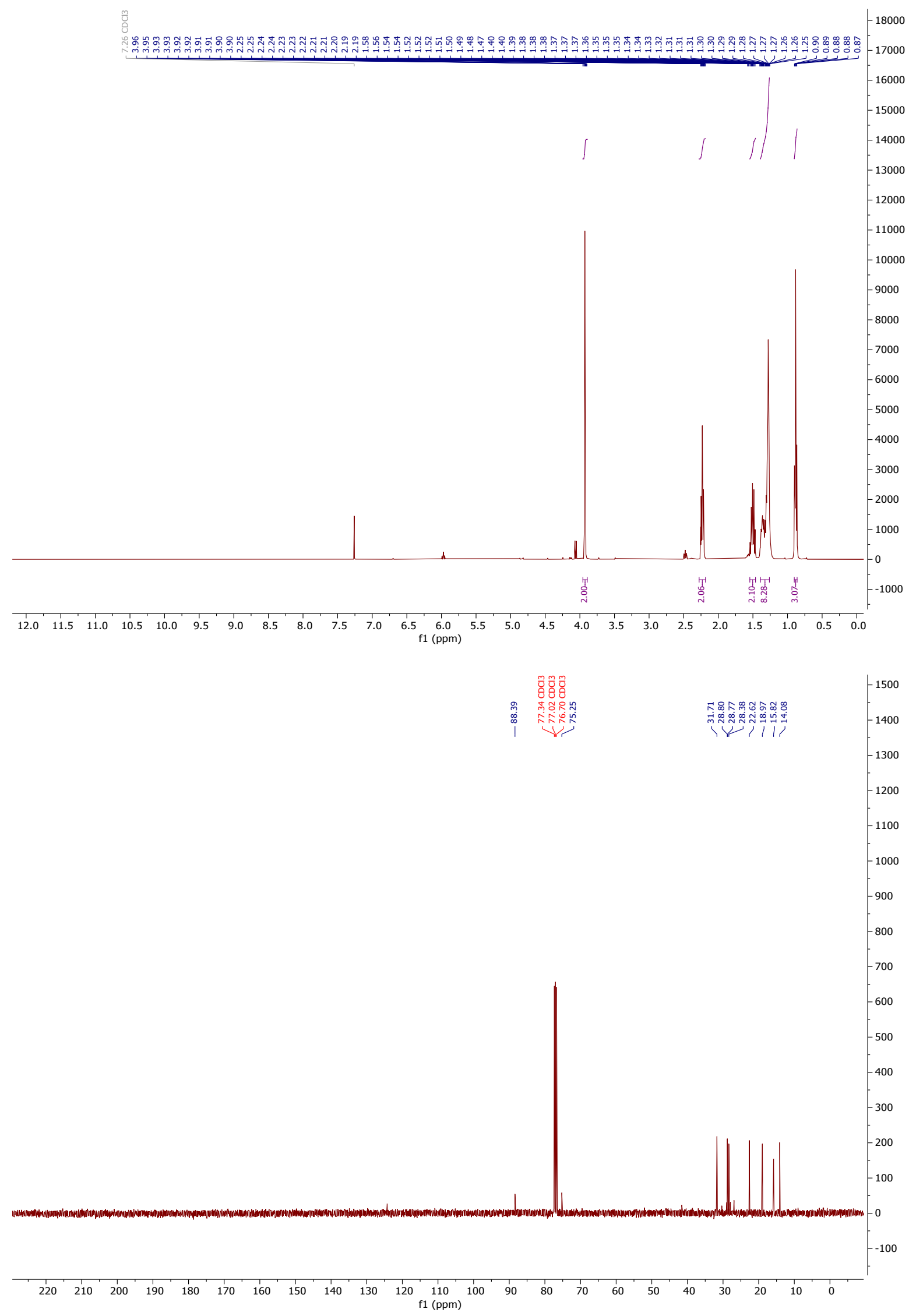


$\curvearrowright \mathrm{Br}$

(E)-(3-bromoprop-1-en-1-yl)benzene $\mathbf{2 7}$

(E)-(3-bromoprop-1-en-1-yl)benzene was synthesised according to catalytic procedure 5 cinnamyl alcohol (153 mg, $1.0 \mathrm{mmol}$ ). ${ }^{1} \mathrm{H}-\mathrm{NMR}$ of the crude mixture showed $91 \%$ conversion to bromide product. The crude reaction mixture was purified by purification method 1 to give the target compound as a pale yellow oil.

Chemical Formula: $\mathrm{C}_{9} \mathrm{H}_{9} \mathrm{Br}$

Molecular Weight: $197.08 \mathrm{gmol}^{-1}$

Yield: $65 \%$ yield (131 $\mathrm{mg}, 0.65 \mathrm{mmol})$

$\delta_{H}(500 \mathrm{MHz}$, Chloroform-d) $7.41-7.37(2 \mathrm{H}, \mathrm{m}), 7.36-7.31(2 \mathrm{H}, \mathrm{m}), 7.30-7.26(1 \mathrm{H}, \mathrm{m}), 6.65(1 \mathrm{H}$, $\mathrm{d}, J$ 15.6), $6.40(1 \mathrm{H}, \mathrm{dt}, J 15.6,7.8), 4.17(2 \mathrm{H}, \mathrm{dd}, J$ 7.8, 1.0).

$\delta_{C}(126 \mathrm{MHz}$, Chloroform-d) $135.8,134.6,128.7,128.4,126.8,125.2,33.5$.

${ }^{1} \mathrm{H}-\mathrm{NMR}$ and ${ }^{13} \mathrm{C}$-NMR were in agreement with the literature. ${ }^{14}$ 

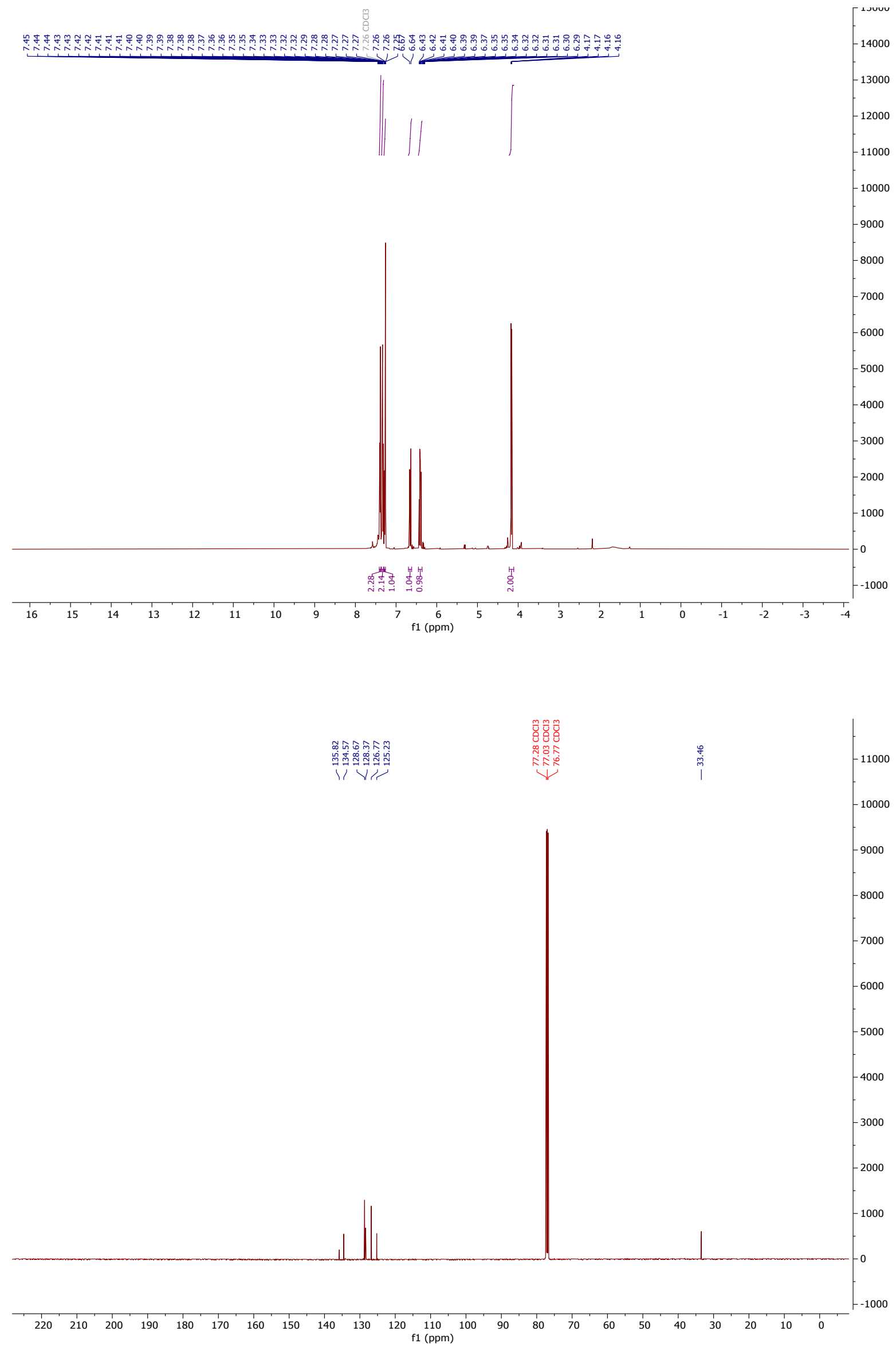
<smiles>BrC(c1ccccc1)c1ccccc1</smiles>

(Bromomethylene)dibenzene $\mathbf{2 8}$

(Bromomethylene)dibenzene was synthesised according to catalytic procedure 5 using diphenylmethanol (184 mg, $1.0 \mathrm{mmol}$ ). ${ }^{1} \mathrm{H}-\mathrm{NMR}$ of the crude mixture showed $>99 \%$ conversion to bromide product. The crude reaction mixture was purified by purification method 1 to give the target compound as a pale yellow oil that slowly solidified on standing to a white solid.

Chemical Formula: $\mathrm{C}_{13} \mathrm{H}_{11} \mathrm{Br}$

Molecular Weight: $247.14 \mathrm{gmol}^{-1}$

Yield: $78 \%$ yield (193 mg, $0.78 \mathrm{mmol})$

$\delta_{H}(500 \mathrm{MHz}$, Chloroform-d) $7.54-7.47(4 \mathrm{H}, \mathrm{m}), 7.37(4 \mathrm{H}, \mathrm{t}, \mathrm{J}$ 7.5), $7.34-7.28(2 \mathrm{H}, \mathrm{m}), 6.33(1 \mathrm{H}, \mathrm{s})$.

$\delta_{c}(126 \mathrm{MHz}$, Chloroform-d) 141.1, 128.6, 128.52 128.2, 55.5

${ }^{1} \mathrm{H}-\mathrm{NMR}$ and ${ }^{13} \mathrm{C}-\mathrm{NMR}$ were in agreement with the literature. ${ }^{7}$ 

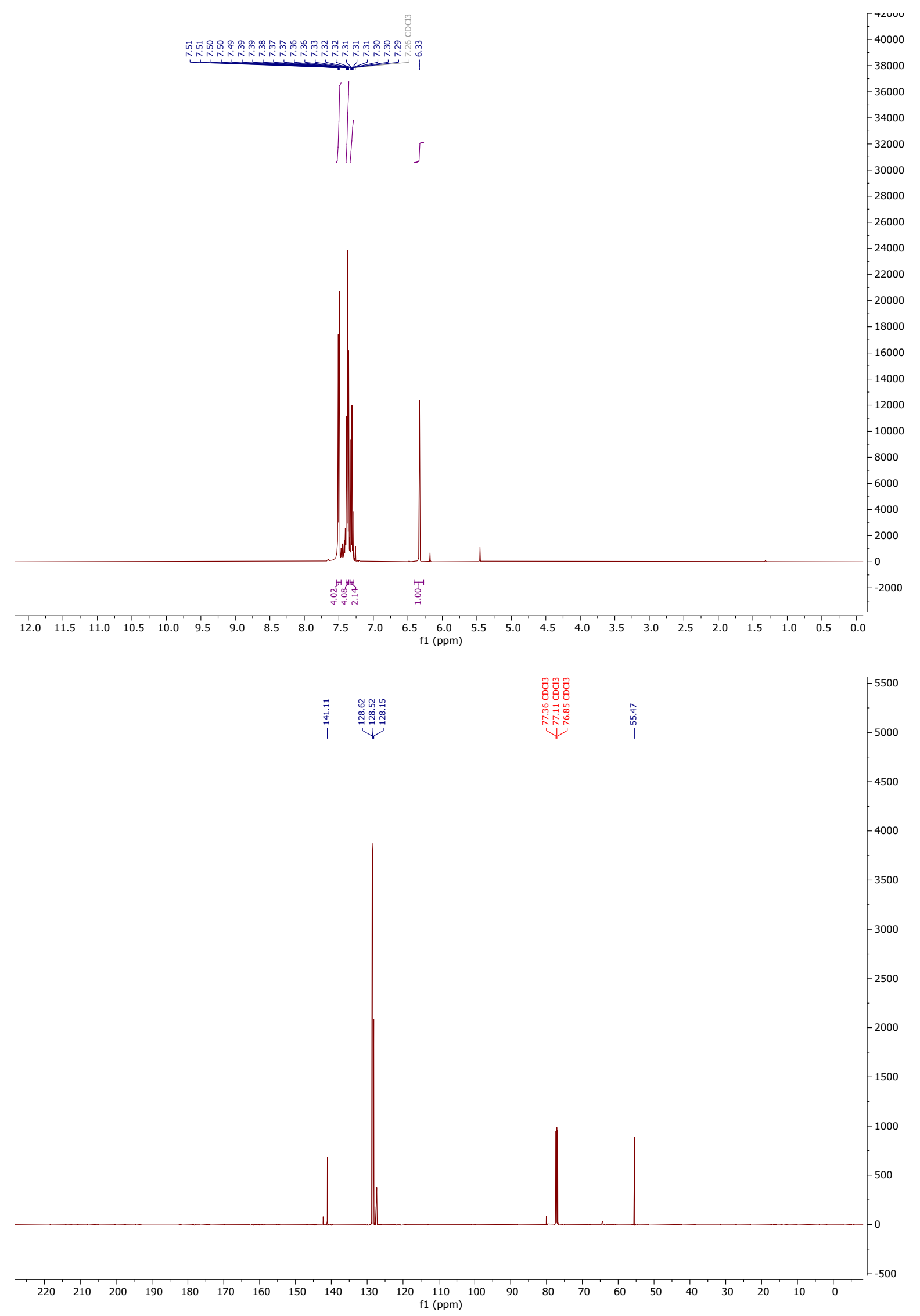
$\square^{\mathrm{Br}}$

3-bromocyclohex-1-ene 29

3-bromocyclohex-1-ene was synthesised according to catalytic procedure 5 using cyclohex-2-en-1-ol (98 mg, $1.0 \mathrm{mmol}$ ). ${ }^{1} \mathrm{H}-\mathrm{NMR}$ and ${ }^{13} \mathrm{C}-\mathrm{NMR}$ of the crude mixture showed $>99 \%$ conversion to product. A small portion was isolated by vacuum distillation for NMR identification.

Chemical Formula: $\mathrm{C}_{6} \mathrm{H}_{9} \mathrm{Br}$

Molecular Weight: $161.04 \mathrm{gmol}^{-1}$

$\delta_{H}(500 \mathrm{MHz}$, Chloroform-d) $5.97-5.90(1 \mathrm{H}, \mathrm{m}), 5.88-5.73(1 \mathrm{H}, \mathrm{m}), 4.89-4.80(1 \mathrm{H}, \mathrm{m}), 2.25-2.13$ $(3 \mathrm{H}, \mathrm{m}), 2.10-2.00(1 \mathrm{H}, \mathrm{m}), 2.00-1.90(1 \mathrm{H}, \mathrm{m}), 1.80-1.61(1 \mathrm{H}, \mathrm{m})$.

$\delta_{c}(126 \mathrm{MHz}$, Chloroform-d) 131.1, 128.9, 49.1, 32.7, 24.7, 18.5.

${ }^{1} \mathrm{H}-\mathrm{NMR}$ and ${ }^{13} \mathrm{C}-\mathrm{NMR}$ were in agreement with the literature. ${ }^{8}$ 


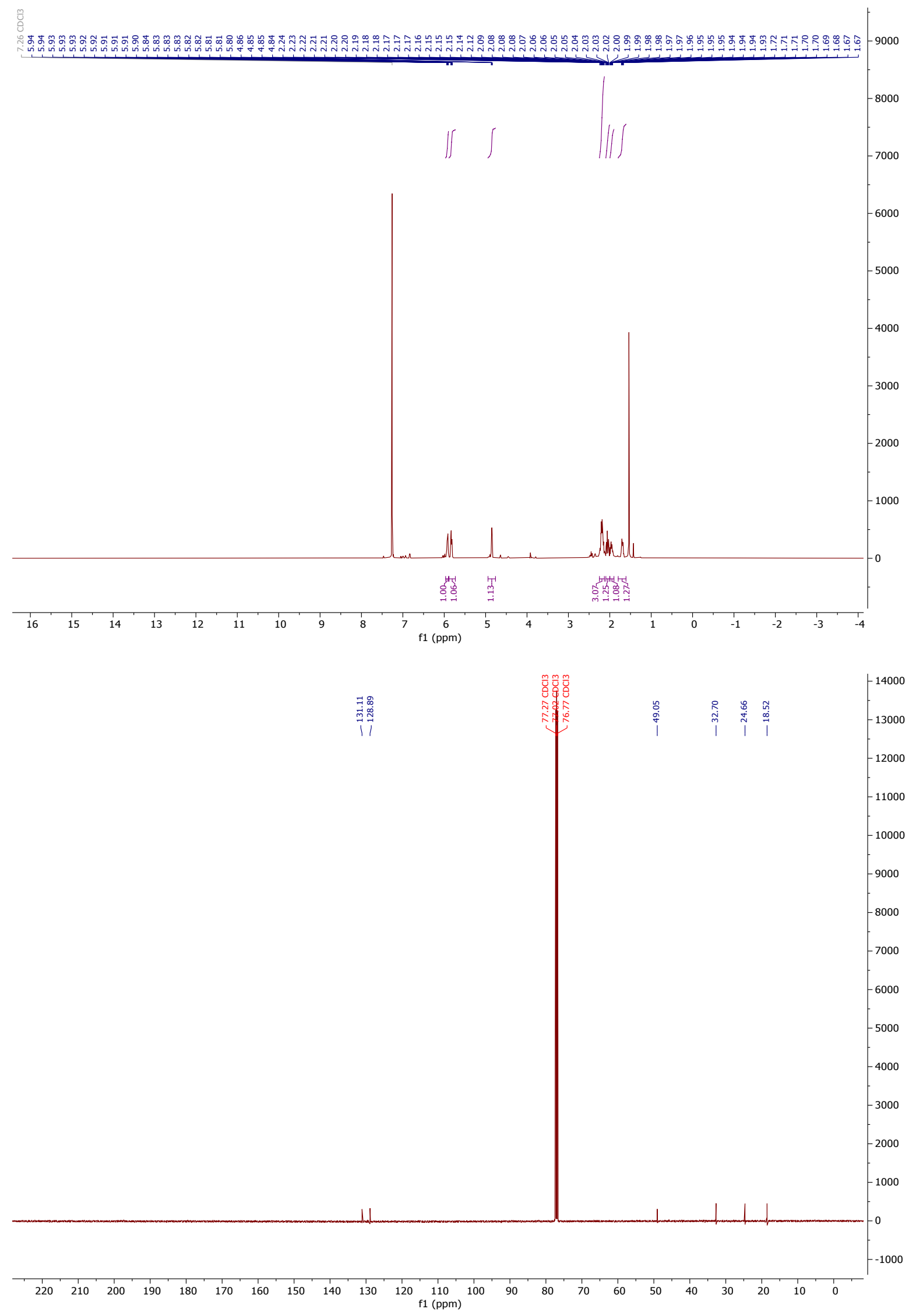


$\overbrace{B r}^{B r}$

1-bromo-4-(1-bromoethyl)benzene 30

1-bromo-4-(1-bromoethyl)benzene was synthesised according to catalytic procedure 5 using 1-(4bromophenyl)ethan-1-ol (100 mg, $0.5 \mathrm{mmol}) .{ }^{1} \mathrm{H}-\mathrm{NMR}$ of the crude mixture showed $88 \%$ conversion to product. The crude reaction mixture was purified by purification method 1 to give the target compound as a pale yellow oil. Yield: $44 \%$ yield $(58 \mathrm{mg}, 0.22 \mathrm{mmol})$.

1-bromo-4-(1-bromoethyl)benzene was also synthesised according to catalytic procedure 5 using 1(4-bromophenyl)ethan-1-ol ( $2.01 \mathrm{~g}, 10.0 \mathrm{mmol})$. The crude reaction mixture was purified according to purification method 3 to give the target compound as a pale yellow oil. Yield: $74 \%$ yield $(1.98 \mathrm{~g}, 7.41$ $\mathrm{mmol})$.

Chemical Formula: $\mathrm{C}_{8} \mathrm{H}_{8} \mathrm{Br}_{2}$

Molecular Weight: $263.96 \mathrm{gmol}^{-1}$

$\delta_{H}(500 \mathrm{MHz}$, Chloroform-d) $7.51-7.42(2 \mathrm{H}, \mathrm{m}), 7.34-7.28(2 \mathrm{H}, \mathrm{m}), 5.15(1 \mathrm{H}, \mathrm{q}, \mathrm{J} 6.9), 2.02(3 \mathrm{H}, \mathrm{d}$, J6.9).

$\delta_{c}(126 \mathrm{MHz}$, Chloroform-d) 142.3, 131.8, 128.5, 122.2, 48.2, 26.7.

${ }^{1} \mathrm{H}-\mathrm{NMR}$ and ${ }^{13} \mathrm{C}-\mathrm{NMR}$ were in agreement with the literature. ${ }^{15}$ 

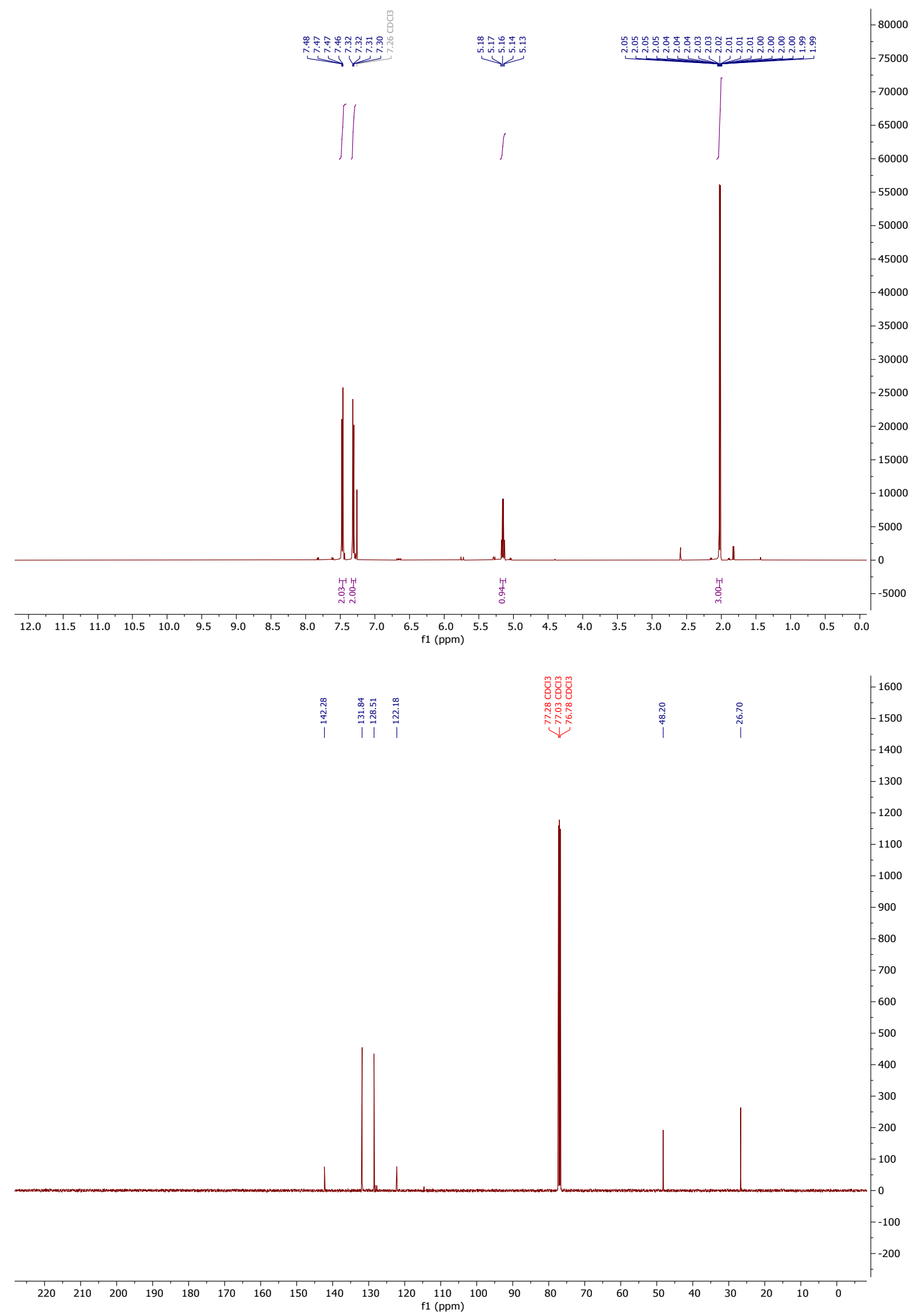
<smiles>O=C(CBr)c1ccccc1</smiles>

2-bromo-1-phenylethan-1-one 31

2-bromo-1-phenylethan-1-one was synthesised according to catalytic procedure 5 using 2-hydroxy-1phenylethan-1-one (136 mg, $1.0 \mathrm{mmol}$ ). HPLC analysis of the crude mixture showed $79 \%$ conversion to product. The crude reaction mixture was purified by purification method 1 to give the target compound as an orange solid. No further purification was carried out.

Chemical Formula: $\mathrm{C}_{8} \mathrm{H}_{7} \mathrm{BrO}$

Molecular Weight: $199.05 \mathrm{gmol}^{-1}$

Yield: 66\% (132 mg, $0.66 \mathrm{mmol})$

$\delta_{H}(500 \mathrm{MHz}$, Chloroform-d) $8.02-7.96(2 \mathrm{H}, \mathrm{m}), 7.65-7.58(1 \mathrm{H}, \mathrm{m}), 7.52-7.48(2 \mathrm{H}, \mathrm{m}), 4.46(2 \mathrm{H}$, s).

$\delta_{\text {c }}(126 \mathrm{MHz}$, Chloroform-d) 191.7, 134.4, 129.3, 129.3, 31.3.

${ }^{1} \mathrm{H}$-NMR and ${ }^{13} \mathrm{C}-\mathrm{NMR}$ were in agreement with the literature. ${ }^{10}$ 


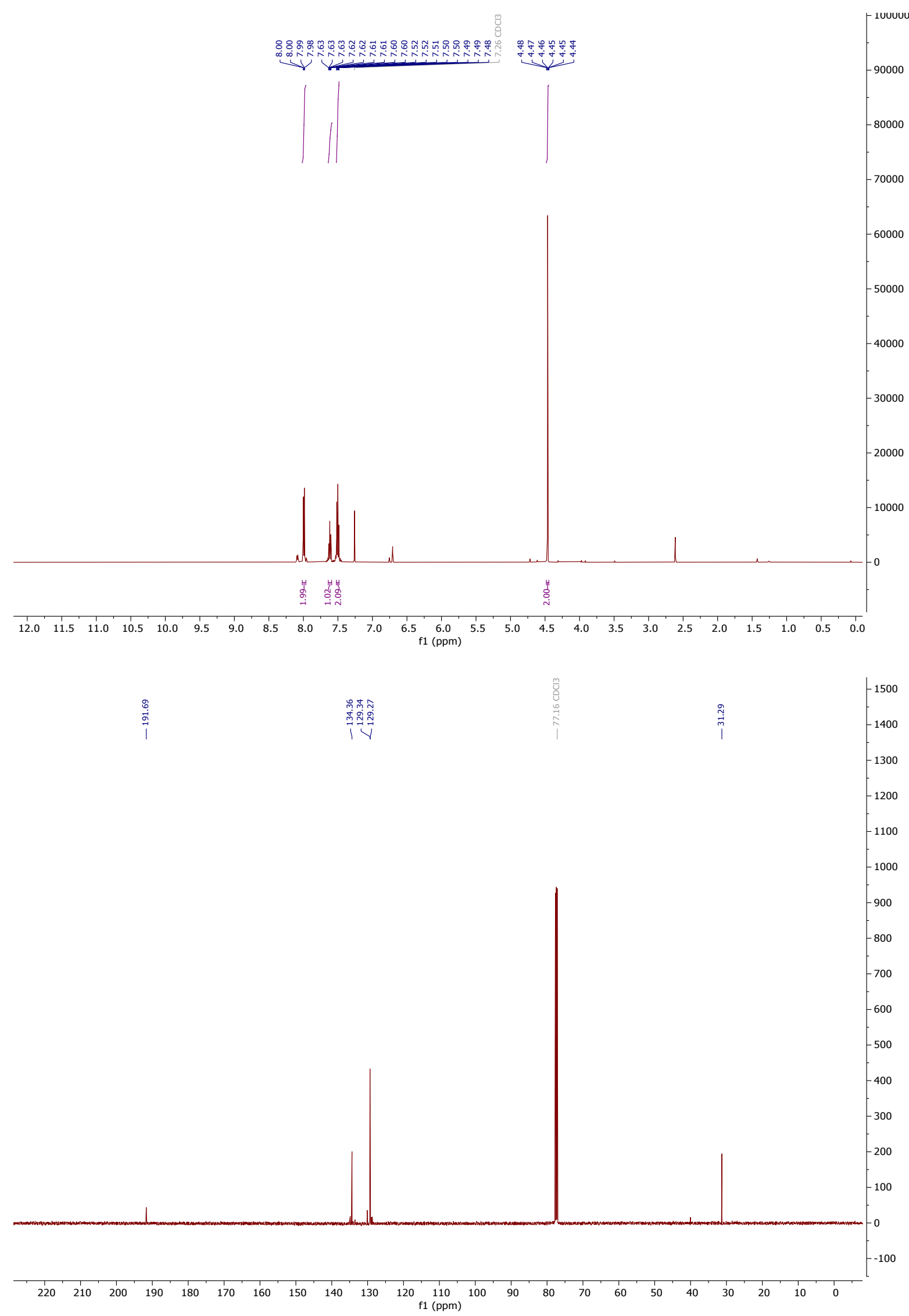




\section{References}

1. Byrne, P. A.; Rajendran, K. V.; Muldoon, J.; Gilheany, D. G., A convenient and mild chromatography-free method for the purification of the products of Wittig and Appel reactions. Org. Biomol. Chem. 2012, 10 (17), 3531-353710.1039/C2OB07074J.

2. Vetter, A. C.; Nikitin, K.; Gilheany, D. G., Long sought synthesis of quaternary phosphonium salts from phosphine oxides: inverse reactivity approach. Chem. Commun. 2018, 54 (46), 5843584610.1039/C8CC02173B.

3. Denton, R. M.; An, J.; Adeniran, B.; Blake, A. J.; Lewis, W.; Poulton, A. M., Catalytic Phosphorus(V)-Mediated Nucleophilic Substitution Reactions: Development of a Catalytic Appel Reaction. J. Org. Chem. 2011, 76 (16), 6749-676710.1021/jo201085r.

4. Longwitz, L.; Jopp, S.; Werner, T., Organocatalytic Chlorination of Alcohols by P(III)/P(V) Redox Cycling. J. Org. Chem 2019, 84 (12), 7863-787010.1021/acs.joc.9b00741.

5. Braddock, D. C.; Pouwer, R. H.; Burton, J. W.; Broadwith, P., Clarification of the Stereochemical Course of Nucleophilic Substitution of Arylsulfonate-Based Nucleophile Assisting Leaving Groups. J. Org. Chem. 2009, 74 (16), 6042-604910.1021/jo900991z.

6. Taguri, T.; Yamakawa, R.; Fujii, T.; Muraki, Y.; Ando, T., Stereospecific inversion of secondary tosylates to yield chiral methyl-branched building blocks, applied to the asymmetric synthesis of leafminer sex pheromones. Tetrahedron: Asymmetry 2012, 23 (11), 85285810.1016/j.tetasy.2012.05.023.

7. Denegri, B.; Streiter, A.; Jurić, S.; Ofial, A. R.; Kronja, O.; Mayr, H., Kinetics of the Solvolyses of Benzhydryl Derivatives: Basis for the Construction of a Comprehensive Nucleofugality Scale. Chem.: Eur. J. 2006, 12 (6), 1648-165610.1002/chem.200500845.

8. Rideau, E.; You, H.; Sidera, M.; Claridge, T. D. W.; Fletcher, S. P., Mechanistic Studies on a CuCatalyzed Asymmetric Allylic Alkylation with Cyclic Racemic Starting Materials. J. Am. Chem. Soc. 2017, 139 (15), 5614-562410.1021/jacs.7b02440.

9. Sasmal, S.; Rana, S.; Lahiri, G. K.; Maiti, D., Manganese-salen catalyzed oxidative benzylic chlorination. J. Chem. Sci. 2018, 130 (7), 8810.1007/s12039-018-1511-7.

10. Xing, Y.; Zhang, M.; Ciccarelli, S.; Lee, J.; Catano, B., Aulll-Catalyzed Formation of $\alpha$-Halomethyl Ketones from Terminal Alkynes. Eur. J. Org. Chem. 2017, 2017 (4), 781-78510.1002/ejoc.201601416.

11. Huy, P. H.; Filbrich, I., A General Catalytic Method for Highly Cost- and Atom-Efficient Nucleophilic Substitutions. Chem. Eur. J. 2018, 24 (29), 7410-741610.1002/chem.201800588.

12. Nguyen, T. V.; Bekensir, A., Aromatic Cation Activation: Nucleophilic Substitution of Alcohols and Carboxylic Acids. Org. Lett. 2014, 16 (6), 1720-172310.1021/ol5003972.

13. Prout, F. S.; Cason, J.; Ingersoll, A. W., Branched-Chain Fatty Acids. V. The Synthesis of Optically Active 10-Methyloctadecanoic Acids. J. Am. Chem. Soc. 1948, 70 (1), 298-30510.1021/ja01181a093.

14. Kroesen, U.; Knauer, L.; Strohmann, C., The Reactivity of Benzyl Lithium Species is Regulated by Intermediate Structures. Angew. Chem. Int. Ed. 2017, 56 (22), 6232-623510.1002/anie.201702377. 15. Bhatthula, B. k. g.; Kanchani, J. r.; Arava, V. r.; Subha, M. C. S., Total synthesis of carbazole alkaloids. Tetrahedron 2019, 75 (7), 874-88710.1016/j.tet.2019.01.003. 